\title{
P INE: Universal Deep Embedding for Graph Nodes via Partial Permutation Invariant Set Functions
}

\author{
Shupeng Gui, Xiangliang Zhang, Pan Zhong, Shuang Qiu, Mingrui Wu, Jieping Ye, Zhengdao Wang, Ji Liu
}

\begin{abstract}
Graph node embedding aims at learning a vector representation for all nodes given a graph. It is a central problem in many machine learning tasks (e.g., node classification, recommendation, community detection). The key problem in graph node embedding lies in how to define the dependence to neighbors. Existing approaches specify (either explicitly or implicitly) certain dependencies on neighbors, which may lead to loss of subtle but important structural information within the graph and other dependencies among neighbors. This intrigues us to ask the question: can we design a model to give the adaptive flexibility of dependencies to each node's neighborhood. In this paper, we propose a novel graph node embedding method (named PINE) via a novel notion of partial permutation invariant set function, to capture any possible dependence. Our method 1) can learn an arbitrary form of the representation function from the neighborhood, without losing any potential dependence structures, and 2) is applicable to both homogeneous and heterogeneous graph embedding, the latter of which is challenged by the diversity of node types. Furthermore, we provide theoretical guarantee for the representation capability of our method for general homogeneous and heterogeneous graphs. Empirical evaluation results on benchmark data sets show that our proposed PINE method outperforms the state-of-the-art approaches on producing node vectors for various learning tasks of both homogeneous and heterogeneous graphs.
\end{abstract}

Index Terms-Graph embedding, partial permutation invariant set function, representation learning

\section{INTRODUCTION}

G RAPH node embedding (or graph node representation learning in some literature |1]) is to learn the numerical representation for each node in a graph by vectors in a Euclidean space, where the geometric relationship reflects the structure of the original graph. Nodes that are "close" in the graph are embedded to have similar vector representations [2]. The learned node vectors benefit a number of graph analysis tasks, such as node classification [3], link prediction [4], community detection [5], recommendation [6], and many others [7].

The graph's neighborhood information is informative enough to define the graph itself. Therefore, the key issue for graph embedding lies on how to model the dependence of each node to its neighbors. Existing approaches mostly specify (either explicitly or implicitly) certain dependencies on neighbors. Deepwalk [8], node2vec [9], and their variants [10], [11] randomly generate a set of paths with a fixed length to learn the representation for each node, which implicitly defines the neighborhood and the dependence among nodes. [12] utilizes the adjacency matrix to represent the neighborhood for every node in a graph and apply matrix factorization for the node embedding learning, which implicitly defines the linear dependence

- S. Gui is with University of Rochester (sgui2@ur.rochester.edu), $X$. Zhang is with King Abdullah University of Science and Technology, Saudi Arabia (Xiangliang.Zhang@kaust.edu.sa)

P. Zhong and Z. Wang are with Iowa State University (pzhong@iastate.edu, zhengdao@iastate.edu),

S. Qiu and J. Ye are with University of Michigan (qiush@umich.edu, jieping@gmail.com),

M. Wu is with Facebook (wu.mingrui@yahoo.com)

J. Liu is with Kwai Inc. (ji.liu.uwisc@gmail.com) among nodes. Neighborhood auto-encoders [13], [14], [15] use a neighborhood vector to represent the neighborhood relations for a node. The neighborhood vector contains a node's pairwise similarity to all the other nodes in a graph. Graph2Gauss [15] embeds each node as a Gaussian distribution based on the graph knowledge. Node2Mixture [16] embeds each node as a mixture of Gaussian distribution to characterize nodes in a comprehensive representation. Deep neural networks for graph representations (DNGR) [14] uses stacked denoising auto-encoder to extract complex non-linear features for each node. Structural Deep Network Embedding method (SDNE) [13] preserves the first-order and second-order proximity for each node in a graph via a semisupervised auto-encoder learning model. Neural network based approaches such as graph convolutional networks (GCN) [17] and GraphSAGE [18] define fixed-depth neural network layers to capture the neighborhood information from one-step neighbors, two-step neighbors, up to $n$-step neighbors and they apply convolution-like functions on these neighbors as the aggregation strategy. Graph attention networks (GATs) [19] and Attention-based Graph Neural Network (AGNN) [20] employ attention mechanism when aggregating the neighbors. Recurrent attention walk [21] and collaborative graph walk 22] explore the neighborhood in reinforcement learning frameworks, which aim to learn enriched node representations for winning the game of node classification.

However, the way of pre-defining (no matter explicitly or implicitly) neighbors and dependence may cause subtle but important loss of structural information within the graph and dependence among neighbors. For example, the family of random walk based methods [8], [9], [10] ignore the 
influence of nodes out of the predefined length to the center node within the path. GCN [17] restricts the form of the dependence on the neighbor nodes to a two-layer aggregation function and inherits considerable complexity from their deep learning lineage [23]. These raise a fundamental question: can we design a model to give the adaptive flexibility of defining dependencies on neighbors?

In this work, we propose a Partial Permutation Invariant Node Embedding method (PINE) by developing a new notion of partial permutation invariant set function, that can

- learn node representations via a universal graph embedding function $f$, without pre-defining pairwise similarity, specifying random walk parameters, or choosing aggregation functions such as element-wise mean, a max-pooling neural network, or long-short term memory units (LSTMs);

- capture the arbitrary permutation invariant relationship of each node to its neighbors;

- be applied to both homogeneous and heterogeneous graphs with complicated types of nodes.

Evaluation results on benchmark data sets show that the proposed PINE outperforms the state-of-the-art approaches on producing node vectors for classification tasks.

Notations: Throughout this paper, we use following notations

- $\mathcal{G}=\{\mathcal{V}, \mathcal{E}\}$ denotes a graph with vertex set $\mathcal{V}$ and edge set $\mathcal{E}$. The corresponding lower case characters $\mathrm{v}$ and $\mathrm{e}$ represents a single vertex and edge.

- $\hat{\mathrm{f}}$ denotes an approximation to a function $\mathrm{f}$.

- $\boldsymbol{P}$ denotes permutation matrix.

- $S_{N}$ denotes a symmetric group.

- $T_{\pi}$ denotes a permutation operator.

- $\sigma(\cdot)$ represents a non-linear activation function.

\section{Related Work}

The main difference among various graph embedding methods lies in how they define the "closeness" between two nodes [2]. First-order proximity, second-order proximity or even high-order proximity have been widely studied for capturing the structural relationship between nodes [24] [25], [26]. Comprehensive reviews of graph embedding can be found in [2], [7], [25], [27]. In this section, we discuss the relevant graph embedding approaches in terms of how node closeness is measured, to highlight our contributions on capturing neighborhood dependency in the most general manner. This section ends up with the review about set functions which are related to the technology we used in this paper.

Matrix Analysis on Graph Embedding: As early as 2011, a spectral clustering method [28] was proposed to take the eigenvalue decomposition of a normalized Laplacian matrix of a graph as an effective approach to obtain an embedding of nodes. Other similar approaches choose different similarity matrices (from the Laplacian matrix) to make a trade-off between modeling the "first-order similarity" and modelling "higher-order similarity" [29], [30], [31]. Node content information can also be fused in the pairwise similarity measure, e.g., in text-associated DeepWalk (TADW) [32], as well as node label information, which results in semi-supervised graph embedding methods, e.g., maxmargin DeepWalk (MMDW) [33]. Recently, an arbitraryorder proximity-preserving graph embedding method is introduced in [12] based on matrix eigen-decomposition, which is applied to a pre-defined high-order proximity matrix. Furthermore, [34] proposes Lanczos network with Lanczos algorithm to construct low rank approximations of the graph Laplacian for graph convolution. For heterogeneous networks, [35] propose a label-involving matrix analysis to learn the classification result of each vertex within a semi-supervised framework.

Random Walk on a Graph to Node Representation: Both deepwalk [8] and node2vec [9] are graph embedding methods to solve the node embedding problem. They convert the graph structures into a sequential context format with random walk [36]. Thanks to the pioneering work of [37] for word representation learning of sentences, deepwalk inherits the learning framework for words representation learning in paragraphs to generate the representation of nodes in random walk context. Then node2vec evolves such the idea with additional hyper-parameters tuning for the trade-off between depth-first search (DFS) and widthfirst search (WFS) to control the direction of random walk. Struc2vec [38] also utilizes the multilayer graph to construct the node representations. [39] proposes a self-paced graph embedding by introducing a dynamic negative sampling method to select difficult negative context nodes in the training process. Planetoid [40] is a semi-supervised learning framework by guiding random walk with available node label information. The heterogeneity of graph nodes is often handled by a heterogeneous random walk procedure [10], or selected relation pairs [41]. The node embeddings of heterogeneous graphs are cast into the hyperbolic spaces in [42]. Authors in [43] consider the predictive text embedding problem on a large-scale heterogeneous text network and the proposed method is also based on pre-defined heterogeneous random walks.

Neighborhood Encoders to Graph Embedding: There are also methods focusing on aggregating or encoding the neighbors' information to generate node embeddings. DNGR [14] and SDNE [13] introduce autoencoders to construct the similarity function between the neighborhood vectors and the embedding of the target node. DNGR defines neighborhood vectors based on random walks and SDNE introduces adjacency matrix and Laplacian eigenmaps to the definition of neighborhood vectors. GraphWave [44] learns the representation of each node's neighborhood via leveraging heat wavelet diffusion patterns. Although the idea of autoencoder brings a great improvement, this type methods are quite expensive in computation when the scale of the graph is up to millions of nodes. As a result, neighborhood aggregation and convolutional encoders are employed to integrate local aggregation for node embedding, such as GCN [17], [45], [46], [47], FastGCN [48], column networks [49], the GraphSAGE algorithm [18], GAT [19]. A recent DRNE [50] method uses layer normalized LSTM to approximate the embedding of a target node by the aggregation of its neighbors' embeddings. Another recent approach named Recurrent Attention Walk (RAW) [21] let an agent make recurrent decisions on next nodes to visit and to aggregate for characterizing the targeting nodes. Following a similar reinforcement learning 
framework, collaborative graph walk [22] selects useful neighbors to aggregate for winning a game of multi-label node classification. And [51] utilizes a set function as a universal approximator to distinguish different graphs with respect to graph classification tasks. For the heterogeneous graphs, HAN [52] constructs a graph neural network by exploiting manually selected meta-paths, and GTN [53] claims it can generate new graph structures to connect those unconnected nodes in the original graph. RHINE [54] handles two types of relations on heterogeneous graph with tailored models to capture the latent structures and semantics of the networks. HetSANN [55] models the transformation between heterogeneous nodes through a projection in lowdimensional entity spaces, and then applies the graph neural network to aggregate multi-relational information of projected neighborhood. The main idea of these methods is involving an iterative or recursive aggregation procedure, e.g., convolutional kernels or pooling procedures to generate the embedding vectors for all nodes, and such aggregation procedures are shared by all nodes in a graph.

Here, we show two GNNs type methods - GCN and GAT - to explain the relation between the GNNs type methods and our PINE framework (5). In the context of node representation, the core representation theorem (Theorem 3.2) in our PINE framework suggests that the output corresponding to node $i$ can be cast into (considering all neighbors of node $i$ are in the same type and itself is a particular type)

$$
\mathbf{x}_{i}^{\prime}=\mathbf{h}\left(\mathbf{g}_{1}\left(\mathbf{x}_{i}\right), \sum_{n \in \mathcal{N}^{i}} \mathbf{g}_{2}\left(\mathbf{x}_{n}\right)\right),
$$

(PINE)

with functions $\mathbf{g}_{1}, \mathbf{g}_{2}$, and $\mathbf{h}$. We will show that both GNN and GAT can be expressed in the form above by appropriately defining $\mathbf{g}_{1}, \mathbf{g}_{2}$, and $\mathbf{h}$. We enumerate the node's dependence functions to its neighbors below: We can construct a general formulation for GCN and GAT, which is

$$
\mathbf{x}_{i}^{\prime}=\boldsymbol{\sigma}\left(\sum_{n \in \mathcal{N}^{i} \cup\{i\}} \alpha_{i, n} \boldsymbol{W} \mathbf{x}_{n}\right),
$$

(GNN)

where

$$
\begin{aligned}
\alpha_{i, n} & =\frac{1}{\left|\mathcal{N}^{i} \cup\{i\}\right|}, \\
\alpha_{i, n} & =\frac{\exp \left(\left[\begin{array}{c}
\boldsymbol{a}_{1} \\
\boldsymbol{a}_{2}
\end{array}\right]^{\top}\left[\begin{array}{c}
\boldsymbol{W} \mathbf{x}_{i} \\
\boldsymbol{W} \mathbf{x}_{n}
\end{array}\right]\right)}{\sum_{k} \exp \left(\left[\begin{array}{c}
\boldsymbol{a}_{1} \\
\boldsymbol{a}_{2}
\end{array}\right]^{\top}\left[\begin{array}{c}
\boldsymbol{W} \mathbf{x}_{i} \\
\boldsymbol{W} \mathbf{x}_{k}
\end{array}\right]\right)},
\end{aligned}
$$

(GCN)

and $\boldsymbol{W}, \boldsymbol{a}_{1}$, and $\boldsymbol{a}_{2}$ are the learnable parameters. The function choice of $\alpha_{i, n}$ in GAT can be complex, but here we only discuss a simplified version of GAT without the non-linear activation function. For GCN, to express the neighborhood aggregation function in the form of Eq. (PINE) we can define

$$
\mathbf{x}_{i}^{\prime}=\mathbf{h}\left(\mathbf{g}_{1}\left(\mathbf{x}_{i}\right), \sum_{n \in \mathcal{N}^{i}} \mathbf{g}_{2}\left(\mathbf{x}_{n}\right)\right),
$$

where we have $\mathbf{g}_{1}(\mathbf{x}):=\boldsymbol{W} \mathbf{z}, \mathbf{g}_{2}(\mathbf{x}):=\left[\begin{array}{c}\boldsymbol{W} \mathbf{z} \\ 1\end{array}\right]$ and $\mathbf{h}\left(\mathbf{z}_{1},\left[\begin{array}{l}\mathbf{z}_{2} \\ z_{3}\end{array}\right]\right):=\boldsymbol{\sigma}\left(\frac{1}{z_{3}+1}\left(\mathbf{z}_{1}+\mathbf{z}_{2}\right)\right)$.
For GAT, the neighborhood aggregation function can be extracted as

$$
\mathbf{x}_{i}^{\prime}=\mathbf{h}\left(\mathrm{g}_{1}\left(\mathbf{x}_{i}\right), \sum_{n \in \mathcal{N}^{i}} \mathbf{g}_{2}\left(\mathbf{x}_{n}\right)\right) .
$$

where $\mathbf{h}\left(z_{1},\left[z_{2}, \mathbf{z}_{3}^{\top}\right]^{\top}\right)=\boldsymbol{\sigma}\left(\frac{\mathbf{z}_{3}}{z_{2}}\right), \mathrm{g}_{1}(\mathbf{x})=\exp \left(\boldsymbol{a}_{1}^{\top} \boldsymbol{W} \mathbf{x}\right)$, and $\mathbf{g}_{2}(\mathbf{x})=\left[\begin{array}{c}\exp \left(\boldsymbol{a}_{2}^{\top} \boldsymbol{W} \mathbf{x}\right) \\ \exp \left(\boldsymbol{a}_{2}^{\top} \boldsymbol{W} \mathbf{x}\right) \boldsymbol{W} \mathbf{z}\end{array}\right]$. We can see that the PINE framework suggests a function structure to characterize a universal dependence, which can be naturally substituted for the dependence function of the existing GNNs methods.

We also noticed the similarity between PINE and GIN [51] which is a recent work. GIN learns the graph embedding by aggregating all the embedding vectors for all nodes in a graph. Therefore, GIN can be utilized to compute the node embedding as well although they emphasize the performance on graph classification empirically. Although both of PINE and GIN study the neighborhood's embedding construction strategy, they are formulated in different problem settings. GIN focuses on solving the Isomorphism of graphs and obtaining the MLP formulation of the embedding generation. PINE considers the universal approximation with a partial permutation invariant function to compute the neighborhood's embedding. Recent works [56], [57] also point out possible general forms of GNN layers.

The above-mentioned methods work differently on how they use neighboring nodes for node embedding. They require pre-defining pairwise similarity measure between nodes, specifying random walk parameters, or choosing aggregation functions. In practice, it usually takes a lot of effort to tune these parameters or try different measures, especially when graphs are complicated with nodes of multiple types, i.e., heterogeneous graphs. This work hence targets on making neighboring nodes play their roles in a most general manner such that their contributions are learned but not user-defined. The resultant embedding method has the flexibility to work on any types of homogeneous and heterogeneous graph.

Our proposed method PINE has a natural advantage on avoiding any manual manipulation of random walking strategies or designs for the relationships between different types of nodes.

Set functions: $[58]$ introduces the notion of set functions as a universal approximator to measure the permutation invariant property of sets but only provides a less rigorous skeleton proof. A very recent work [59] further improves the theoretical analysis on invariant maps by neural networks. The notion of partial permutation invariant set functions proposed in this paper is a more generic version of the set function. We find a neater form than [59] even in the special case and also provide rigorous proofs for the representation theorem.

\section{THE PROPOSED PINE FRAMEWORK}

In this section, we first formally define the problem, and then introduce a new definition - partial permutation invariant set function. This section ends up with the proposed PINE framework whose key is the representation theorem of the partial permutation invariant set function. 
We target on designing graph embedding models for general graphs that may include $K$ different types of nodes ( $K=1$ corresponds to the homogeneous graphs and $K \geq 2$ corresponds to heterogeneous graphs). Formally, a graph $\mathcal{G}=\{\mathcal{V}, \mathcal{E}\}$, where the node set $\mathcal{V}=\bigcup_{k=1}^{K} \mathcal{V}_{k}$, i.e., $\mathcal{V}$ is composed of $K$ disjoint types of nodes. One instance of such a graph is the academic publication network, which includes different types of nodes for papers, publication venues, author names, author affiliations, research domains etc. Given such a graph $\mathcal{G}$, our goal is to learn the embedding vector for each node in this graph.

We use $\mathrm{x}^{\mathrm{v}} \in \mathbb{R}^{d}$ to denote the representation of node $\mathrm{v}$. The node $\mathrm{v} \in \mathcal{V}_{k}$ can be represented by its neighbors' embedding vectors via a function $\mathbf{f}$

$$
\mathbf{x}^{\mathrm{v}}=\mathbf{f}\left(\boldsymbol{X}_{1}^{\mathrm{v}}, \boldsymbol{X}_{2}^{\mathrm{v}}, \cdots, \boldsymbol{X}_{K}^{\mathrm{v}}\right),
$$

where $\boldsymbol{X}_{k}^{\mathrm{v}}$ is a matrix with column vectors corresponding to the embedding of node v's neighbors in type $k . \boldsymbol{X}_{k}^{\mathrm{v}}$ could also be the representation vectors associating with node $v^{\prime}$ s type- $k$ neighbors. We use $d$ to denote the dimensions of the embedding vector. Note that the way we have defined the function $\mathbf{f}$ implies that it is node dependent. For learning to be possible, the embedding functions for different nodes will share common parameters, as will become clear in Section 3.2

\subsection{Partial permutation invariant set functions}

The graph's neighborhood information is informative enough to define the graph itself. Therefore, the key to defining the graph embedding lies in how to model the dependence of each node to its neighbors, that is, what function $\mathbf{f}$ in Eq. (1) to choose. Most existing approaches only (either explicitly or implicitly) stress on some specific forms to characterize the dependence between each node and its neighbors while ignoring other potential dependence.

We propose a universal graph embedding model that does not pre-define the dependence form between each node and its neighbors due to the key observation: all neighboring nodes reachable from a target node $\mathrm{v}$ are not distinguishable from the view of the target node if they belong to the same type. To formally define the function satisfying this property, we introduce a new notation named partial permutation invariant set function.

Definition 3.1. [Partial permutation invariant set function] Given a space $\mathbb{W}:=\mathbb{W}_{1} \times \mathbb{W}_{2} \cdots \times \mathbb{W}_{K}$, a continuous real valued map $\mathbf{f}: \mathbb{W} \longrightarrow \mathbb{R}^{d}$ is partially permutation invariant if

$$
\mathbf{f}\left(\boldsymbol{X}_{1} \boldsymbol{P}_{1}, \boldsymbol{X}_{2} \boldsymbol{P}_{2}, \cdots, \boldsymbol{X}_{K} \boldsymbol{P}_{K}\right)=\mathbf{f}\left(\boldsymbol{X}_{1}, \boldsymbol{X}_{2}, \cdots, \boldsymbol{X}_{K}\right)
$$

for all permutation matrices $\boldsymbol{P}_{k}$ and $k \in[K]$.

This definition essentially requires the function value of $\mathbf{f}(\cdot)$ to be invariant to swapping any two columns of $\boldsymbol{X}_{k}$.

\subsection{PINE: the representation of partial permutation in- variant set function}

Unfortunately, this function is not simply learnable because the permutation property is hard to guarantee directly. One straightforward idea to represent the partial permutation invariant set function is to define it in the following form

$$
\begin{aligned}
& \mathbf{x}^{\mathrm{v}}=\mathbf{f}\left(\boldsymbol{X}_{1}^{\mathrm{v}}, \cdots, \boldsymbol{X}_{K}^{\mathrm{v}}\right) \\
& :=\sum_{\boldsymbol{P}_{1} \in \mathbb{P}_{\left|\mathcal{V}_{1}^{v}\right|}} \sum_{\boldsymbol{P}_{\mathbf{2}} \in \mathbb{P}_{\left|\mathcal{V}_{2}^{\mathrm{v}}\right|}} \cdots \sum_{\boldsymbol{P}_{K} \in \mathbb{P}_{\left|\mathcal{V}_{K}^{v}\right|}} \mathbf{t}\left(\boldsymbol{X}_{1}^{\mathrm{v}} \boldsymbol{P}_{1}, \cdots, \boldsymbol{X}_{K}^{\mathrm{v}} \boldsymbol{P}_{K}\right),
\end{aligned}
$$

where $\mathcal{V}_{k}^{\mathrm{v}}$ denotes type- $k$ neighbors of node $\mathrm{v}$ and $\mathbb{P}_{\mid \mathcal{V}_{k}^{\mathrm{v}}}$ denotes the set of $\left|\mathcal{V}_{k}^{\mathrm{v}}\right| \times\left|\mathcal{V}_{k}^{\mathrm{v}}\right|$ permutation matrices for any $k \in[K], \boldsymbol{X}_{k}^{\mathrm{v}} \boldsymbol{P}_{k}$ is to permute the columns in $\boldsymbol{X}_{k}^{\mathrm{v}}$, and $\mathbf{t}(\cdot)$ is a properly designed function. It is easy to verify that the function defined in Eq. (4) is partial permutation invariant, but it is intractable because it involves $\prod_{k=1}^{N}\left(\left|\mathcal{V}_{k}^{\mathrm{v}}\right| !\right)$ "sum" items. Our solution of learning function $\mathrm{f}$ is then based on the following important theorem, which gives a neat and general way to represent any partial permutation invariant set function.

Theorem 3.2. [Representation theorem of partial permutation invariant set functions] Let $\mathbf{f}$ be a continuous real-valued function defined on a compact set with the following form

$$
\mathbf{f}(\underbrace{\mathbf{x}_{1,1}, \mathbf{x}_{1,2}, \cdots, \mathbf{x}_{1, N_{1}}}_{G_{1}}, \underbrace{\mathbf{x}_{2,1}, \cdots, \mathbf{x}_{2, N_{2}}}_{G_{2}}, \cdots, \underbrace{\mathbf{x}_{K, 1}, \cdots, \mathbf{x}_{K, N_{K}}}_{G_{K}}),
$$

where $\mathbf{x}_{k, n} \in \mathbb{R}^{M_{k}}$. If function $\mathbf{f}$ is partial permutation invariant, that is, any permutations of the elements within the group $G_{k}$ for any $k$ does not change the function value, then there must exist functions $\mathbf{h}(\cdot)$ and $\left\{\mathbf{g}_{k}(\cdot)\right\}_{k=1}^{K}$ to approximate $\mathbf{f}$ with arbitrary precision in the following form

$$
\mathbf{f}(\cdot)=\mathbf{h}\left(\sum_{n=1}^{N_{1}} \mathbf{g}_{1}\left(\mathbf{x}_{1, n}\right), \sum_{n=1}^{N_{2}} \mathbf{g}_{2}\left(\mathbf{x}_{2, n}\right), \cdots, \sum_{n=1}^{N_{K}} \mathbf{g}_{K}\left(\mathbf{x}_{K, n}\right)\right) .
$$

The rigorous proof is provided in Appendix A.2 This result suggests a neat but universal way to represent any partial permutation invariant set function. For instance, a popular permutation invariant set function widely used in deep learning $\max (\cdot)$ can be approximated with an arbitrary precision by

$$
\max \left(x_{1}, x_{2}, \cdots, x_{N}\right) \approx \mathrm{h}\left(\sum_{i=1}^{N} \mathrm{~g}\left(x_{i}\right)\right)
$$

with $\mathrm{g}(z)=[\exp (k z) \cdot z, \exp (k z)]$, and $\mathrm{h}\left(\left[z, z^{\prime}\right]\right)=z / z^{\prime}$, as long as $k$ is large enough. This is because

$$
\begin{aligned}
& \max \left(x_{1}, x_{2}, \cdots, x_{N}\right)=\lim _{k \rightarrow \infty} \mathrm{h}\left(\sum_{i=1}^{N} \mathrm{~g}\left(x_{i}\right)\right) \\
& =\lim _{k \rightarrow \infty}\left(\sum_{i=1}^{N} \exp \left(k x_{i}\right)\right)^{-1} \sum_{i=1}^{N} \exp \left(k x_{i}\right) \cdot x_{i} .
\end{aligned}
$$

Theorem 3.2 only establishes the existence of the approximation. To obtain concrete forms of $\mathbf{h}(\cdot)$ and $\mathbf{g}_{k}(\cdot)^{\prime}$ 's, one can always use three-layer fully connected neural networks (Input, hidden, output) to approximate it (to any precision) [60], [61|. For example, $\mathbf{h}(\mathbf{z})=\boldsymbol{B} \boldsymbol{\sigma}(\boldsymbol{A z}+\boldsymbol{a})+\boldsymbol{b}$. Such an intuition leads us to make the approximation of Eq. (5) with a five-layer neural network (input, three hidden layers, output). The following theorem shows that we can even choose a simpler and neater form for $\mathbf{h}(\cdot)$ and $\mathbf{g}_{k}(\cdot)^{\prime}$ s to approximate 
an arbitrary $\mathbf{f}(\cdot)$ as a whole than simply approximating Eq. (5) with a five-layer neural network. More specifically, we can formulate $\mathbf{f}(\cdot)$ for $\mathbf{h}(\cdot)$ and $\mathbf{g}_{k}(\cdot)$ 's as a whole with four-layer neural network, which is enough for the universality. For simplicity, we consider the case that the image of $\mathbf{h}(\cdot)$ or $\mathbf{f}(\cdot)$ is one dimension. The case with a high dimensional image can be simply applied based on the one dimensional case.

Theorem 3.3. The functions $\mathbf{h}(\cdot)$ and $\mathbf{g}_{k}(\cdot)$ 's in Theorem 3.2 can be chosen in the following form (assuming that the image of $\mathbf{h}(\cdot)$ is one dimension) to obtain the approximation function $\hat{\mathbf{f}}(\cdot)$ for $\mathbf{f}(\cdot)$ in Theorem 3.2 .

$$
\begin{gathered}
\mathbf{h}\left(\left[\mathbf{z}_{1}^{\top}, \cdots, \mathbf{z}_{K}^{\top}\right]^{\top}=: \overline{\mathbf{z}} \mid \boldsymbol{c}, \boldsymbol{W}, \boldsymbol{b}\right)=\boldsymbol{c}^{\top} \boldsymbol{\sigma}(\boldsymbol{W} \overline{\mathbf{z}}+\boldsymbol{b}), \\
\mathbf{g}\left(\mathbf{x} \mid T, \boldsymbol{u},\left\{\boldsymbol{a}_{t}, \boldsymbol{b}_{t}^{\prime}\right\}_{t=1}^{T}\right)=\left[\begin{array}{c}
\boldsymbol{\sigma}\left(\left(\boldsymbol{u} \otimes \boldsymbol{a}_{1}\right) \mathbf{x}+\boldsymbol{b}_{1}^{\prime}\right) \\
\boldsymbol{\sigma}\left(\left(\boldsymbol{u} \otimes \boldsymbol{a}_{2}\right) \mathbf{x}+\boldsymbol{b}_{2}^{\prime}\right) \\
\vdots \\
\boldsymbol{\sigma}\left(\left(\boldsymbol{u} \otimes \boldsymbol{a}_{T}\right) \mathbf{x}+\boldsymbol{b}_{T}^{\prime}\right)
\end{array}\right],
\end{gathered}
$$

where $\boldsymbol{\sigma}(\cdot)$ is the element-wise squashing activation function, and we omit the subscript $k$ all hyper-parameters of $\mathbf{g}_{k}(\cdot)$.

Beside that it implies a four-layer (other than a five-layer) neural network is sufficient to approximate an arbitrary $\mathbf{f}(\cdot)$, this theorem suggests a much neater form to approximate $\mathbf{f}(\cdot)$ in Eq. (5) by sharing parameters and restricting the parameter space.

Remark 3.4. Let us take a close look at the form of $\mathbf{g}(\cdot)$ in Theorem 3.3 Note that $\boldsymbol{U}_{t}:=\boldsymbol{u} \otimes \boldsymbol{a}_{t}$ is a rank one matrix. If we simply apply the generic neural network approximation theorem, $\boldsymbol{U}_{t}$ should be a free matrix. Therefore, here we actually reduce the number of parameters by explicitly restricting it within the rank one matrix space. In addition, the $\boldsymbol{u}$ component in all $\boldsymbol{U}_{t}$ 's are shared, which partially further reduces the number of parameters.

To visualize the form of $\hat{\mathbf{f}}(\cdot)$ based on the construction in Theorem 3.3. we provide two figures for two cases: Homogeneous Graph (nodes do not have types; in other words, all nodes belong to the same type) and Heterogeneous Graph (K-types nodes) in Fig. 1 and Fig. 2 respectively.

The objective function for the neural network parameter optimization depends on applications. As one example, a least square cost function of the embedded vectors $\mathrm{x}^{\mathrm{v}}$ and the embedding output as provided by the neural network can be minimized for consistency. This is a joint optimization problem: both the embedding vectors $\mathbf{x}^{\mathrm{v}} \mathrm{s}$ and the embedding functions are jointly optimized. The numerical optimization algorithm and complexity are similar to those for standard deep neural networks. In a semi-supervised setting, it is also possible to incorporate a supervised component into the objective function; see numerical examples in section 4 and specifically Eq. 6.

\section{EMPIRICAL EXPERIMENTS STUdY}

In the section, we validate and report the performance of the proposed partial permutation invariant set function theorem on various aspects of graph node embedding learning tasks comparing to state-of-the-art algorithms: (1) to evaluate the applicability of PINE on embedding problem of general graphs, we conduct experiments on both homogeneous and heterogeneous graphs; (2) we also visualize the embedding vectors obtained by PINE to $2 \mathrm{D}$ space via t-SNE with respect to the true and predicted labels; and (3) for ablation study, we investigate the impact of hyper-parameters for PINE and show the performace on two datasets, Cora and Wikipedia. More experimental results of ablation study are provided in Appendix B.

\subsection{Evaluation on Homogeneous Graphs}

First we consider the multi-class node classification problem over the homogeneous graphs. Given a graph with partially labeled nodes, the goal is to learn the representation for each node for predicting the class for unlabeled nodes. To fulfill the learning requirements, we have a basic assumption that the embedding of an arbitrary node in the graph can be calculated via PINE with the neighborhood as the input. For short, we denote the PINE embedding function to aggregate neighborhood information for a node v by $\mathbf{x}^{\mathrm{v}}=\hat{\mathbf{f}}\left(\mathcal{X}^{\mathrm{v}}\right):=\left[\hat{f}_{1}\left(\mathcal{X}^{\mathrm{v}}\right), \hat{f}_{2}\left(\mathcal{X}^{\mathrm{v}}\right), \cdots, \hat{f}_{d}\left(\mathcal{X}^{\mathrm{v}}\right)\right]^{\top} \in \mathbb{R}^{d}$, where $\mathcal{X}^{\mathrm{v}}:=\left(\boldsymbol{X}_{1}^{\mathrm{v}}, \boldsymbol{X}_{2}^{\mathrm{v}}, \cdots, \boldsymbol{X}_{K}^{\mathrm{v}}\right)$ contains all embeddings of neighbors of v. Since this section evaluates the homogeneous graphs, the $K$ in all $\hat{f}(\cdot)$ s is set to 1 . To fulfill the requirement of a specific learning task, we propose an overall learning model with PINE by involving an unsupervised component and a supervised component at the same time

$$
\begin{aligned}
\min _{\substack{\left\{\mathbf{x}^{\mathrm{v}}\right\}_{\mathrm{v} \in \mathcal{V}},\left\{\hat{f}_{m}\right\}_{m=1}^{d}, \theta}} \frac{1}{\lambda|\mathcal{V}|} \sum_{\mathrm{v} \in \mathcal{V}} \| \mathbf{x}^{\mathrm{v}}- & {\left[\hat{f}_{1}\left(\mathcal{X}^{\mathrm{v}}\right), \cdots, \hat{f}_{d}\left(\mathcal{X}^{\mathrm{v}}\right)\right]^{\top} \|^{2} } \\
& +\frac{1}{\left|\mathcal{V}_{\text {label }}\right|} \sum_{\mathrm{v} \in \mathcal{V}_{\text {label }}} \ell_{\theta}\left(\mathbf{x}^{\mathrm{v}}, \boldsymbol{y}^{\mathrm{v}}\right) .
\end{aligned}
$$

The first term of the objective in Eq. (6) is the unsupervised learning component, which restricts the representation error between the target node and its neighbors with $L_{2}$ norm since it is allowed to have noise in a practical graph. The second term is the supervised component, which is flexible to be replaced with any designed learning task on the nodes in a graph. For example, to a regression problem, a least square loss can be chosen to replace $\ell_{\theta}(\cdot)$ and a cross entropy loss can be used to formulate a classification problem.

The details of PINE for multi-class case are as follows: (1) Supervised Component: Softmax function is chosen to formulate our supervised component in Eq. (6). For an arbitrary embedding $\mathbf{x} \in \mathbb{R}^{d}$, we have the probability term as $\mathrm{P}(y=i \mid \mathbf{x})=\frac{\exp \left(\boldsymbol{w}_{i}^{\top} \mathbf{x}+b_{i}\right)}{\sum_{j=1}^{C} \exp \left(\boldsymbol{w}_{j}^{\top} \mathbf{x}+b_{j}\right)}$ for predicting $\mathbf{x}$ with class $i$, where $\boldsymbol{w}_{i} \in \mathbb{R}^{d}$ and $b_{i} \in \mathbb{R}$ are classifier parameters for class $i$, and $C$ is the number of classes. Therefore, the supervised component in Eq. (6) is formulated as $\frac{1}{\mid \mathcal{V}_{\text {label }}} \sum_{\mathrm{v} \in \mathcal{V}_{\text {label }}} \sum_{i=1}^{C}\left[-y_{i}^{\mathrm{v}} \log \mathrm{P}\left(y^{\mathrm{v}}=i \mid \mathbf{x}^{\mathrm{v}}\right)\right]+$ $\lambda_{w} \sum_{i=1}^{C} \operatorname{Reg}\left(\boldsymbol{w}_{i}\right)$, where $y_{i}^{\mathrm{v}} \in\{0,1\}$ is the true label for training, $\operatorname{Reg}\left(\boldsymbol{w}_{i}\right)$ is an $L_{2}$ regularization for $\boldsymbol{w}_{i}$, and $\lambda_{w}$ is chosen to be $10^{-3}$; (2) Unsupervised embedding mapping Component: The balance hyper-parameter $\lambda_{1}$ is set to be 0.005 . We follow the formulation in Theorem 3.3 and $c \in \mathbb{R}^{16}$, $T=32$, and $\boldsymbol{u} \in \mathbb{R}^{16}$. We apply an ADAM algorithm to compute the effective solutions for the learning variables simultaneously.

Datasets: We evaluate the performance of PINE and other methods for comparison on five benchmark datasets: 


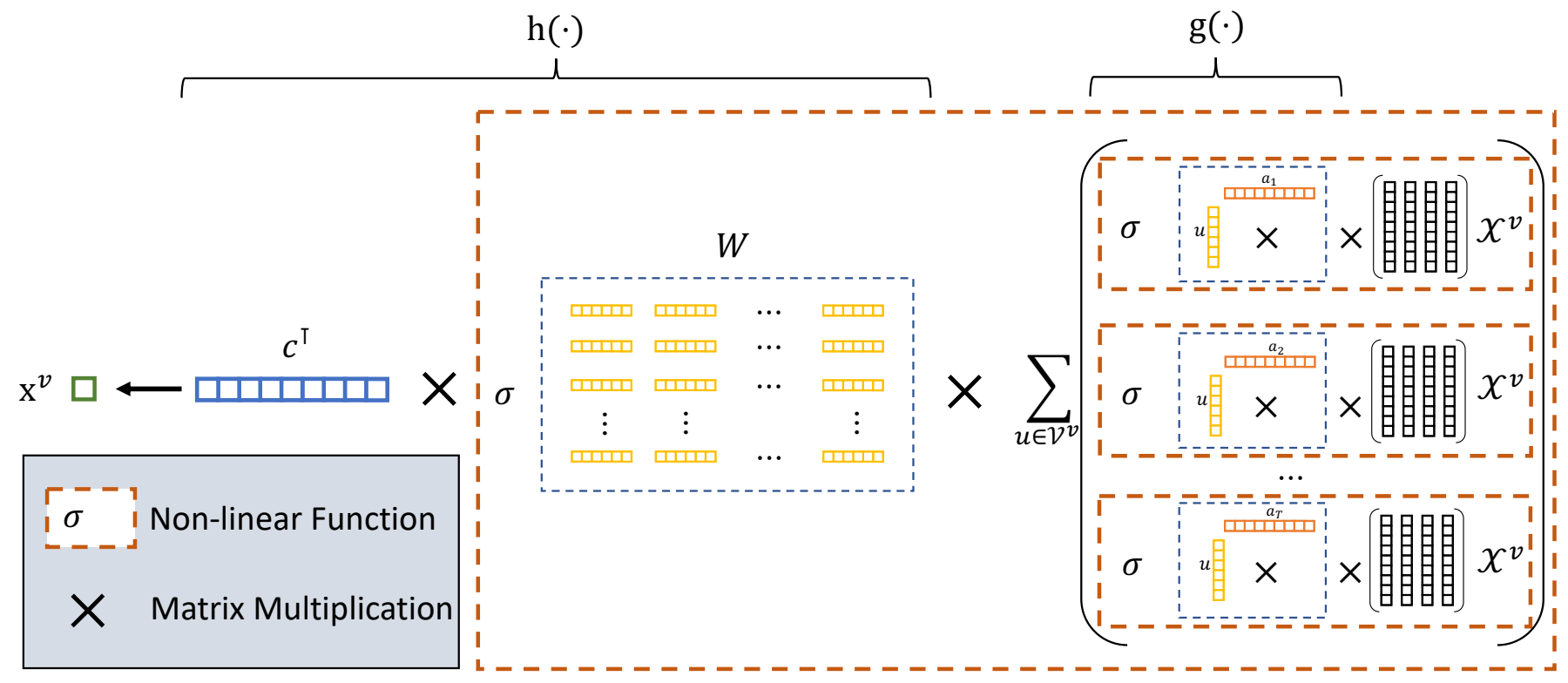

Fig. 1: Illustration of $f(\cdot)$ for "Homogeneous" Graph Node Embedding Learning using Theorem 3.3 (There is only 1 type of nodes.) For an arbitrary node $\mathrm{v}$, the neighborhood information $\mathcal{X}^{\mathrm{v}}$ is aggregated via the function $\mathbf{h}$ and $\mathbf{g}$ to compute the embedding of node $\mathrm{v}$. Since there is only 1-type node neighbors, the function $\mathrm{g}$ are shared for all neighbors.

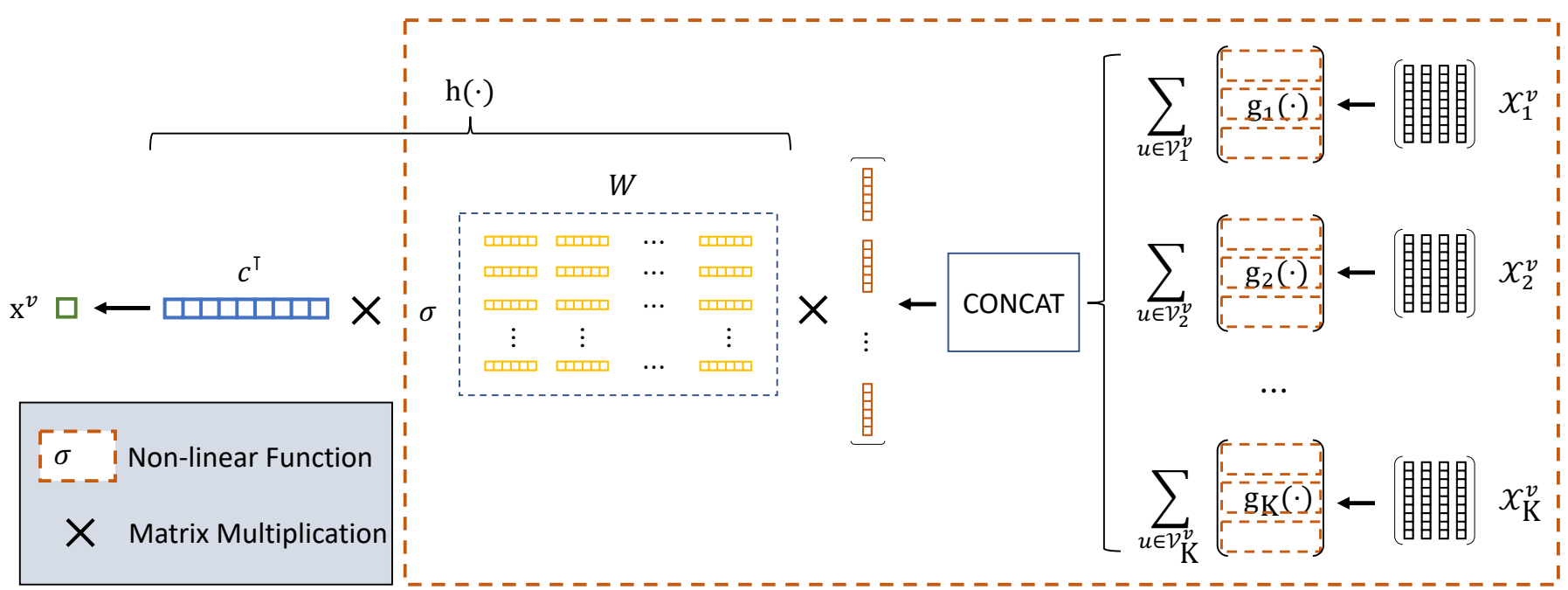

Fig. 2: Illustration of $\mathbf{f}(\cdot)$ for "Heterogeneous" Graph Node Embedding Learning using Theorem 3.3 (There are $\mathrm{K}$ types of nodes). $\left\{\mathbf{g}_{1}, \cdots, \mathbf{g}_{K}\right\}$ are dealing with $K$-type node neighborhood respectively. The outputs of $\left\{\mathbf{g}_{1}, \cdots, \mathbf{g}_{K}\right\}$ are concatenated and $\mathbf{h}$ extracts the heterogeneous neighborhood information to formulate the embedding of node $\mathrm{v}$.

TABLE 1: Summary of Datasets

\begin{tabular}{cccccc|cc}
\hline & CORA & Citeseer & PUbMed & WiKipedia & EMAIL-EU & DBLP & BlOGCATALOG \\
\hline \#NODE & 2,708 & 3,312 & 19,717 & 2,405 & 1,005 & $27 K+3.7 K$ & $55,814+5,413$ \\
\#EDGE & 5,429 & 4,732 & 88,651 & 17,981 & 25,571 & $338,210+66,832$ & $1.4 M+619 \mathrm{~K}+343 \mathrm{~K}$ \\
\hline \#CLASSES & 7 & 6 & 3 & 17 & 42 & 4 (MULTI-LABEL) & 5 (MULTI-LABEL) \\
\hline
\end{tabular}

Cora [62], Citeseer [63], Pubmed [64], Wikipedia [64], and Email-eu [65]. The details of these five datasets are presented in Table 1

Baseline methods: To evaluate the learning capability of PINE, we compare it with baseline algorithms listed below:

- Deepwalk [8] is an unsupervised graph embedding method which relies on the random walk and word2vec method. For each vertex, we take 80 random walks with length 40 , and set window size as 10 . Since deepwalk is unsupervised, we apply a logistic regression on the generated embeddings for node classification.

- Node2vec [9] is an improved graph embedding method 
based on deepwalk. We set the window size as 10 , the walk length as 80 and the number of walks for each node is set to 100. Similarly, node2vec is unsupervised as well. We apply the same evaluation procedure on the embeddings of node2vec as what we did for deepwalk.

- Struc2vec [38] chooses the window size as 10, the walking length as 80 , the number of walks from each node as 10, and 5 iterations in total for SGD.

- GraphWave [44] chooses the heat coefficient as 1000, the number of characteristic functions as 50 , the number of Chebyshev approximations as 100, and the number of steps as 20.

- WYS (Watch-Your-Step) [66] chooses the learning rate as 0.2 , the highest power of normalized adjacency matrix as 5 , the regularization coefficient as 0.1 , and uses the "Log Graph Likelihood" as objective function.

- MMDW [33] is a semi-supervised learning framework of graph embedding which combines matrix decomposition and SVM classification. We tune the method multiple times and take 0.01 as the hyper-parameter $\eta$ in the method which is recommended by the authors.

- Planetoid [40] is a semi-supervised learning framework. We set the batch size as 200, learning rate as 0.01 , the batch size for label context loss as 200, and mute the node attributes as input while using softmax for the model output.

- GCN (Graph Convolutional Networks) [17] chooses the convolutional neural networks into the semisupervised embedding learning of graph. We eliminate the node attributes for fairness as well.

- GAT (Graph Attention Networks) [19] choose the learning rate as 0.005 , the coefficient of the regularization as 0.0005 , and the number of hidden units as 64 . To make the comparison fair, we mute the node attributes in the training of GATs as well.

Experiment setup and results. For a fair comparison, the dimension of representation vectors is chosen to be the same for all algorithms (the dimension is 64). The hyperparameters are fine-tuned for all of them. More experiment environment and comparison details are presented in the Appendix.

In this multi-class classification scenario, we use Accuracy as the evaluation criterion. The percentage of labeled nodes is chosen from $10 \%$ to $90 \%$ and the remaining nodes are used for evaluation. All experiments are repeated for five times and we report the mean and standard deviation of the performance of each graph embedding method in Figure 3 We can observe that in most cases, PINE outperforms other methods and in few cases, PINE performs the second best behind of MMDW. With the analysis of the experiment results, we expect to answer several questions: Q1) How powerful is PINE on the practical datasets? Q2) How does PINE work compared with Matrix Analysis, Random Walk, and Graph Neural Network based methods? For the first question, it is observed that PINE outperforms other methods in most cases stated in Figure 3 PINE gains general capability to multiple real-world graph datasets on the node classification task. To the question 2, different methods adopt various learning strategies. The method MMDW also applies the semi-supervised learning framework which combines the matrix decomposition with the supervised classification. It performs well in most datasets except for the Email-eu. However, PINE performs well on all of them. Although MMDW adopts the semi-supervised learning strategy which learns from the knowledge with the node classification labels, there is still limitation on the matrix analysis side compared to our partial permutation invariant function. For the comparison with graph neural networks, GCN performs the third best in most cases. Due to the exclusion of node features, GCN and GAT have downgraded performance. Especially, GAT without using node features even has worse performance than the unsupervised node embedding learning methods. For those random-walk based methods, most of them are constructed under the unsupervised learning scenario. It is reasonable that they performed worse than other supervised or semi-supervised learning methods. Similar to the case of GAT, planetoid doesn't perform well though it also takes the node classification into consideration during the training process. In addition, comparing to these GNN methods, PINE optimizes graph node embedding vectors and neural netowrk parameters jointly. To draw a brief conclusion, PINE has the flexibility to adopt the general graph structures in the learning of node embeddings compared to other existing graph node embedding learning methods.

In addition to the reported accuracy under the graph node classification task. We also present the visualization of graph node embedding in the 2D space with the t-SNE method. The results of Cora, Citeseer, Email-eu, Pubmed, and Wikipedia are presented in Fig 4, Fig 5, Fig 6, Fig 7, and Fig 8 respectively. We present all the figures with the ratio of unlabeled nodes as $50 \%$ for the node classification task. For each figure, we illustrate the t-SNE results with respect to the true labels and the predicted ones on test set. As what we expected, we can observe that the embedding vectors can be easily clustered with t-SNE for both true and predicted labels. There is the difference between the true label figure and predicted label one due to the difference of true and predicted labels.

Then, we provide several figures to show the hyperparameter sensitivity for PINE. We take a case study on the sensitivity to the embedding dimensions on Cora and Wikipedia dataset. The results are shown in Figure 9. For dimensions of $8,16,32$, and 64 , we run the experiments for 5 times with the ratio of unlabeled nodes as $50 \%$ for the node classification task and compute the mean and standard deviation of the accuracy results for GCN, MMDW, and PINE. As shown in the illustration, the performance rises along the increasing of the dimension of the representations for nodes for GCN, MMDW, and PINE, and PINE always achieves higher performance.

Exploration under graph neural network framework In addition to the experiments on the comparison of the graph node classification task with our overall model in Eq. (6), we also explore the potential of partial permutation invariant function in the existing Graph Neural Network (GNN). With the study of existing GNNs, we find that they rely on some specific aggregation function to measure the relationship between each target node and its neighborhood. It reminds us of substituting the neighborhood aggregation component with PINE in existing graph neural network (GNN) e.g., GraphSAGE [18] and GAT [19], and evaluate 

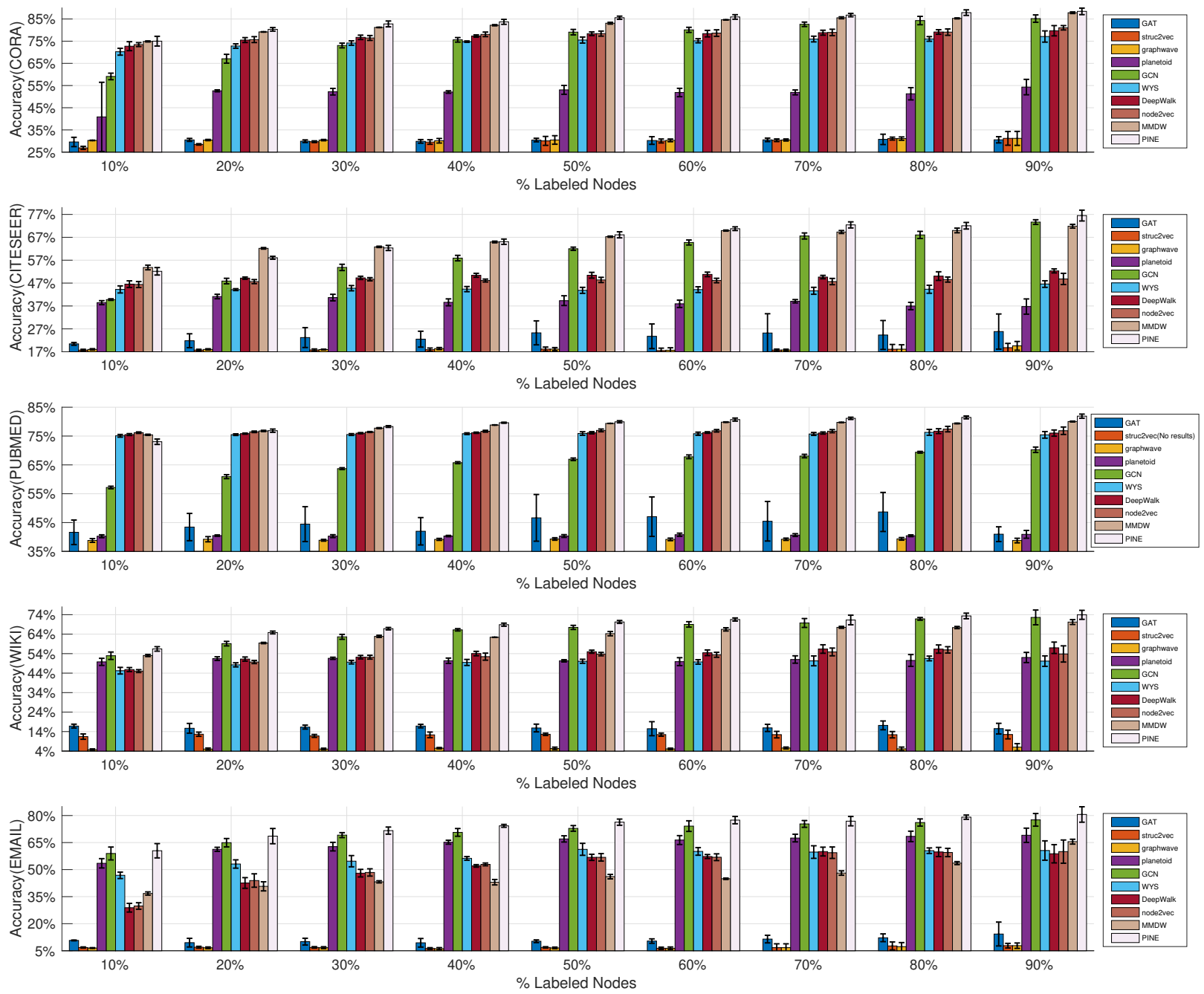

Fig. 3: Accuracy (\%) of multi-class classification in Cora, Citeseer, Pubmed, Wikipedia, and Email-eu dataset

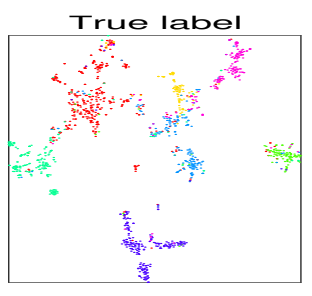

True label

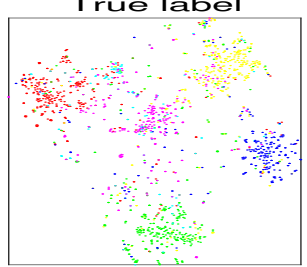

Fig. 5: The t-SNE of Citeseer dataset.
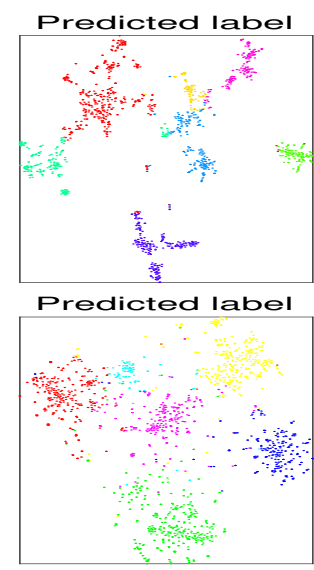
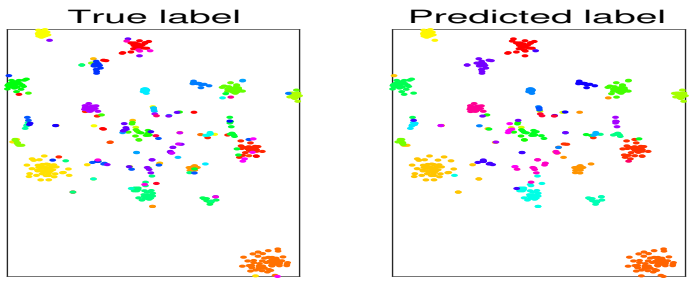

Fig. 6: The t-SNE of Email-eu dataset.
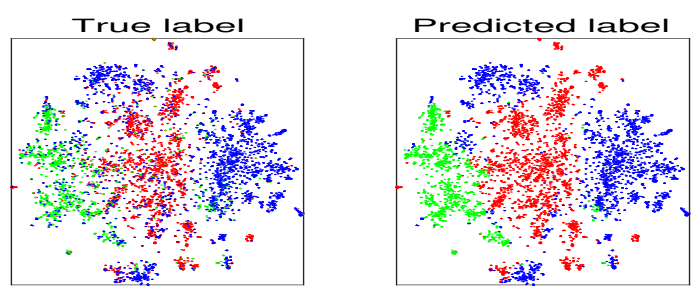

Fig. 7: The t-SNE of Pubmed dataset. its capability on neighborhood aggregation. It is expected that PINE under the GNN framework performs better than the orignal GraphSAGE [18] and GAT [19]. Here we relax the assumption of Theorem 3.2 a little. Since graph neural networks adopt layer-stacks design, the node embeddings are computed through layers. Therefore, we alter the relation between target node embedding and its neighborhood to be

$$
\mathbf{x}_{\mathrm{v}}^{(l+1)}=\mathbf{h}\left(\sum_{n=1}^{N_{1}} \mathbf{g}_{1}\left(\mathbf{x}_{1, n}^{(l)}\right), \sum_{n=1}^{N_{2}} \mathbf{g}_{2}\left(\mathbf{x}_{2, n}^{(l)}\right), \cdots, \sum_{n=1}^{N_{k}} \mathbf{g}_{k}\left(\mathbf{x}_{k, n}^{(l)}\right)\right),
$$



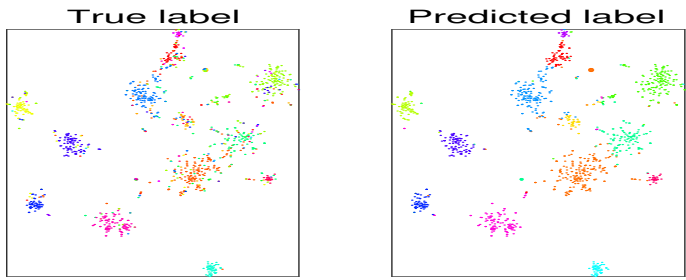

Fig. 8: The t-SNE of Wikipedia dataset.

where $l$ denotes the layer number, and $\mathbf{x}^{(l)}$ is the hidden output in the graph neural network. Hence, the $(l+1)$ th layer's target node embedding is computed by the neighborhood node embeddings in $l$-th layer. We adopt the PINE function (7) as the aggregation function adopted in graph neural networks such as GraphSage and GAT. Other graph neural network settings are maintained as the same adopted in the original GNNs. We validate PINE on two datasets. (1) Dataset PPI is a set of protein-protein interaction graphs, which poses an inductive learning problem. We take this chance to validate PINE by learning from 20 graphs with another 2 graphs for validation, and then classifying nodes in 2 other different graphs into 121 classes. We compare PINE with GraphSAGE and GAT on this task. The results in Table 2 show that PINE has higher classification accuracy on the nodes in the previously unseen graphs. That justifies the superior performance of PINE in inductive setting. (2) Reddit [18] is a large dataset including 232,965 nodes. We validate PINE on this dataset to see its generalizability on learning from old data to predict new data. We use the first 20 days' data as the training set and the rest split up into the validation $(30 \%)$ and test set $(70 \%)$, which should be classified with multi-labels by choosing as accurate as possible from 50 labels. Table 3 shows that PINE has the best performance on this challenging task. Overall, we can conclude that PINE can be a suitable aggregator in GNN framework without subtle aggregation structure design.

Extension of PINE on unsupervised graph node representation Learning. For the learning case without labels (e.g., node ranking), the unsupervised term in Eq. (6) could lead to the trivial solution (all 0 's embedding for all nodes). To avoid the trivial solution in the unsupervised case, we can simply add some virtual supervised information by encouraging the dissimilarity if two nodes are not connected or far away from each other. Specifically, we can replace the second term by

$$
\sum_{(\mathrm{u}, \mathrm{v}) \in \mathcal{U}} \frac{\left\langle\mathbf{x}^{\mathrm{v}}, \mathrm{x}^{\mathrm{u}}\right\rangle}{\left\|\mathrm{x}^{\mathrm{v}}\right\| \cdot\left\|\mathrm{x}^{\mathrm{u}}\right\|}
$$

where $\mathcal{U}$ is a predefined set of pair nodes far away enough from each other. In the case when we have labels for some nodes, we can add (8) as an additional term.

\subsection{Comparison on heterogeneous graphs}

We next conduct evaluation on heterogeneous graphs, where the learned node embedding vectors are used for multi-label classification. Since multiple types of nodes are presented in heterogeneous graphs, we substitute the unsupervised embedding mapping component with $\sum_{k=1}^{K} \frac{1}{\lambda_{k}\left|\mathcal{V}_{k}\right|} \sum_{\mathrm{v} \in \mathcal{V}_{k}}\left\|\mathbf{x}^{\mathrm{v}}-\left[\hat{f}_{1}\left(\mathcal{X}^{\mathrm{v}}\right), \cdots, \hat{f}_{d}\left(\mathcal{X}^{\mathrm{v}}\right)\right]^{\top}\right\|^{2}$.
The supervised component in a multi-label setting can be addressed by formulating a set of binary classification problem (one for each label). Therefore, (1) Supervised Component: we apply logistic regression for each instance $x$ and its $i$-th label $y_{i}$ via letting $\bar{\ell}\left(\mathbf{x}, y_{i}\right)=\log \left(1+\exp \left(\boldsymbol{w}_{i}^{\top} \mathbf{x}+b_{i}\right)\right)-y_{i}\left(\boldsymbol{w}_{i}^{\top} \mathbf{x}+\right.$ $\left.b_{i}\right)$, where $\boldsymbol{w}_{i} \in \mathbb{R}^{d}$ and $b_{i} \in \mathbb{R}$ are classifier parameters for the $i$-th label. Then, defining $y_{i}^{\mathrm{v}} \in\{0,1\}$ to be the true label for training, the supervised component in (6) is formulated as $\frac{1}{\mathcal{V}_{\text {label }}} \sum_{\mathbf{v} \in \mathcal{V}_{\text {label }}} \sum_{i=1}^{C} \bar{\ell}\left(\mathbf{x}^{\mathbf{v}}, y_{i}^{\mathrm{v}}\right)+\lambda_{w} \sum_{i=1}^{C} \operatorname{Reg}\left(\boldsymbol{w}_{i}\right)$, where $C$ is the number of labels, $\operatorname{Reg}\left(\boldsymbol{w}_{i}\right)$ is the regularization term for $\boldsymbol{w}_{i}$, and $\lambda_{w}$ is chosen as $10^{-4}$; (2) Unsupervised Embedding Mapping Component: The balance hyper-parameter $\left[\lambda_{1}, \lambda_{2}\right]$ is set to be $[0.2,200]$. The hyper-parameters are $c \in \mathbb{R}^{8}$, $T=16$, and $\boldsymbol{u} \in \mathbb{R}^{8}$.

Datasets: The applied datasets include: $\mathbf{D B L P} \mid 67$ is an academic community network. Here we obtain a subset of the large network with two types of nodes, authors and key words from authors' publications. The generated subgraph includes $27 K$ (authors) $+3.7 K$ (key words) vertexes. The link between a pair of author indicates the coauthor relationships, and the link between an author and a word means the word belongs to at least one publication of this author. There are 66,832 edges between pairs of authors and 338,210 edges between authors and words. Each node can have multiple labels out of four. BlogCatalog [68] is a social media network with 55,814 users and according to the interests of users, they are classified into multiple overlapped groups. We take the five largest groups to evaluate the performance of methods. Users and tags are two types of nodes. The 5,413 tags are generated by users with their blogs as keywords. Therefore, tags are shared with different users and also have connections since some tags are generated from the same blogs. The number of edges between users, between tags and between users and tags are about $1.4 \mathrm{M}, 619 \mathrm{~K}$ and $343 \mathrm{~K}$, respectively. Each user is associated with multiple labels out of five. The total number of labels is five and due to the multilabel classification setting, each user may have several possible labels.

Baseline Methods: To illustrate the valid performance of PINE on heterogeneous graphs, we conduct the experiments on two stages: (1) comparing PINE with Deepwalk $[\overline{8}]$ and node2vec [9] on the graphs by treating all nodes as the same type (PINE with $K=1$ in a homogeneous setting); (2) comparing PINE with the state-of-the-art heterogeneous graph embedding method, metapath2vec [10], in a heterogeneous setting. The hyper-parameters of the method are fine-tuned and metapath2vec++ is chosen as the option for the comparison.

Experiment Setup and Results: For the datasets DBLP and BlogCatalog, we conduct the experiments on each of them and compare the performance among all methods mentioned above. Since it is a multi-label classification task, we take F1-score (macro, micro) as the evaluation metrics for the comparison. The users in BlogCatalog or authors in DBLP work are classification targets. We vary the ratio of labeled nodes from $10 \%$ to $90 \%$, repeat all experiments for five times and report the mean and standard deviation of their performance in the Figure 10.

We can observe that in most cases, PINE in heterogeneous setting has the best performance. Comparing with the 

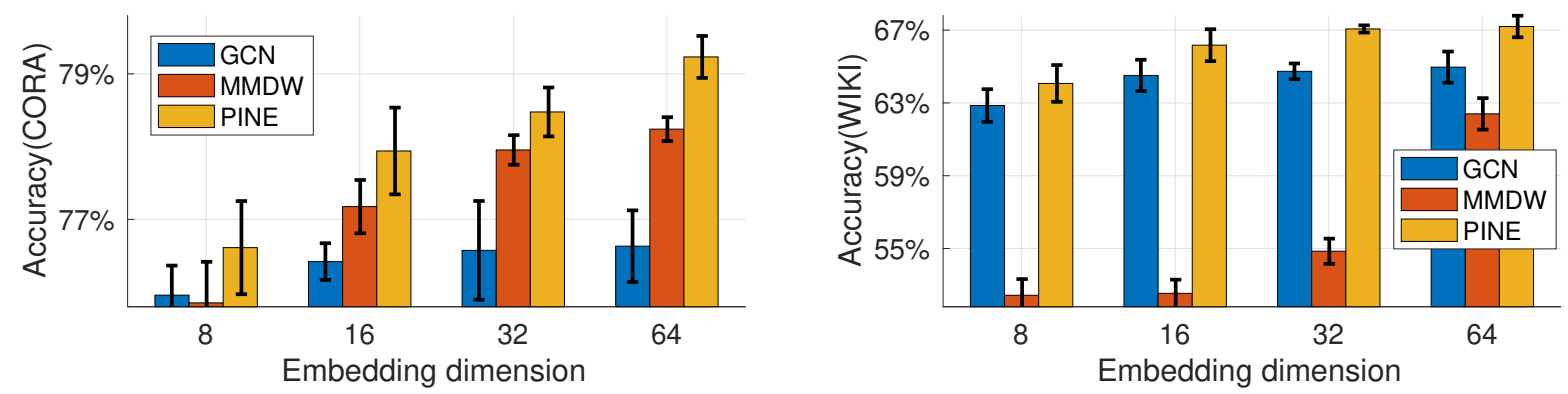

Fig. 9: Dimension sensitivity illustration for PINE on Cora and Wikipedia.

TABLE 2: Inductive prediction results for the PPI dataset (micro-averaged F1 scores)

\begin{tabular}{|c|c|c|c|c|c|}
\hline METHODS & GRAPHSAGE-POOL $[\overline{18}]$ & PINE & GRAPHSAGE $[\overline{19}]$ & GAT $|\overline{19}|$ & PINE \\
\hline RESULTS & 0.600 & 0.637 & $0.768=$ & $0.97 \overline{3}$ & 0985 \\
\hline
\end{tabular}

TABLE 3: Prediction results for the Reddit dataset (micro-averaged F1 scores)

\begin{tabular}{c|cccc}
\hline METHODS & DEEPWALK & DEEPWALK+FEATURES & GRAPHSAGE-POOL $[18]$ & PINE \\
\hline RESULTS & 0.324 & 0.691 & 0.949 & $\mathbf{0 . 9 5 1}$ \\
\hline
\end{tabular}
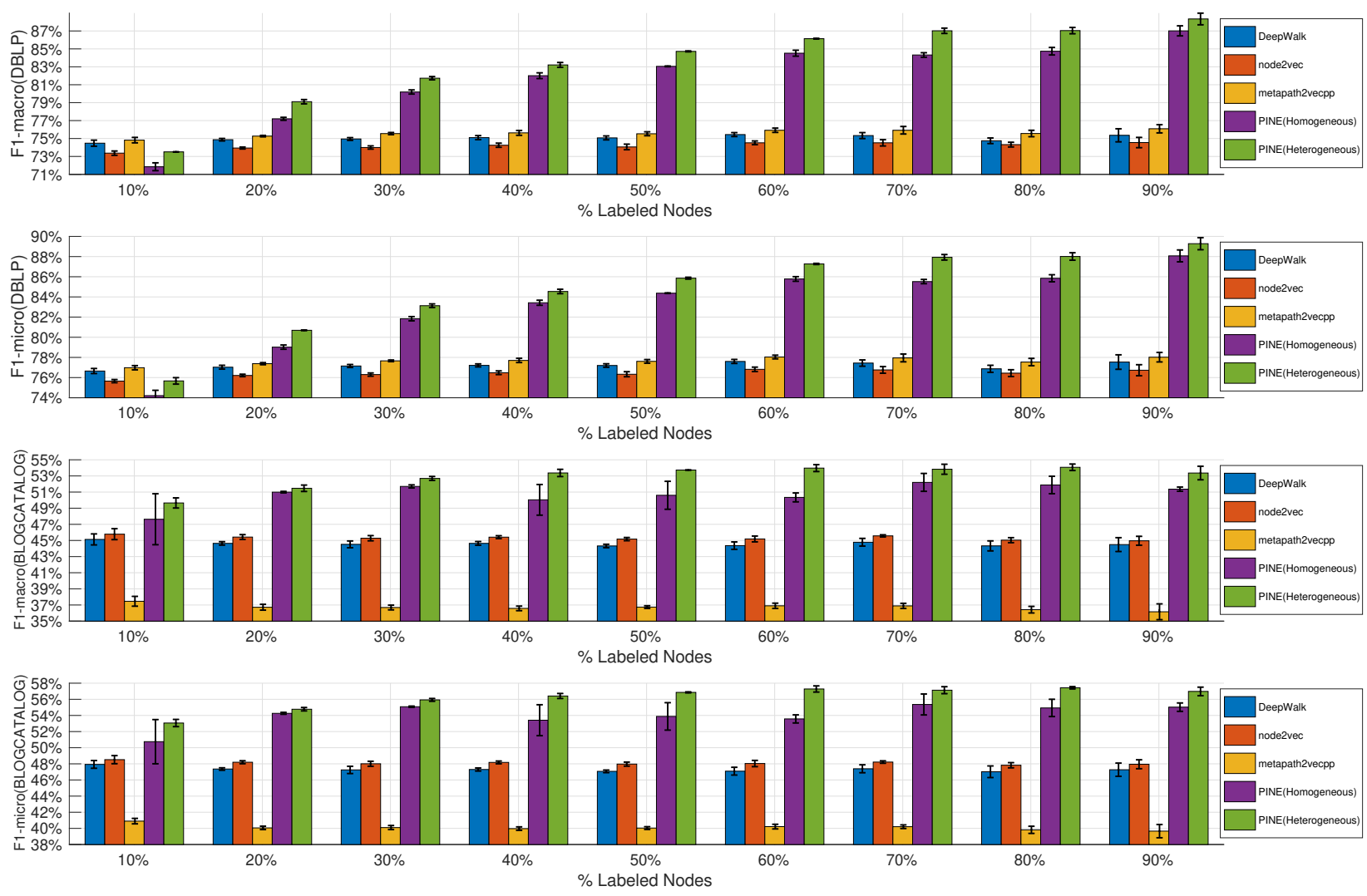

Fig. 10: F1-score (macro, micro) (\%) of multi-label classification in heterogeneous graphs

PINE implemented in homogeneous setting, PINE in heterogeneous setting adopts the advantage of multi-relations setting and also maintain the general neighborhood structure learning ability. PINE in homogeneous setting is better than deepwalk and node2vec in the same homogeneous setting, and is even better than metapath2vec++ in heterogeneous setting (achieving the second best results). Overall, the superior performance of PINE in Figure 3 and 10 demonstrates the validity of our proposed universal graph embedding mechanism.

Case Study: PINE on heterogeneous graph node embedding learning under graph neural network setting 
TABLE 4: Prediction results on the heterogeneous graph node classification task (F1 scores)

\begin{tabular}{|c|c|c|c|c|c|c|c|}
\hline & DEEPWALK [53] & METAPATH2VEC [53] & GCN [53] & GAT [53] & HAN [53] & GTN [53] & PINE \\
\hline ACM & 67.42 & 87.61 & $91.6 \overline{0}$ & $92.3 \overline{3}$ & 90.96 & 92.68 & 92.52 \\
\hline IMDB & 32.08 & 35.21 & 56.89 & 58.14 & 56.77 & 60.92 & 59.91 \\
\hline
\end{tabular}

In the following, we take some efforts on the exploration of PINE on the heterogeneous graphs node embedding learning performance under the graph neural network settings. For the existing heterogeneous graph neural network learning methods, they were supposed to design specific graph neural network structure to obtain the graph node embedding with respect to the specific learning tasks, such as heterogeneous graph node classification problem. Therefore, we want to show that PINE is capable to fit an arbitrary graph neural network structure without any specific design, but just replacing the graph neural network layers with the PINE functions. In the comparison, we utilize two benchmark datasets, ACM, and IMDB. These heterogeneous graph datasets were introduced in Graph Transformer Networks (GTN) [53] to show the performance on heterogeneous graph node classification performance. For the ACM, it is a citation network. The learning goal is to distinguish the categories of papers which are presented as nodes in the graph. The graph dataset contains 3 types of nodes and node features for each node in the graph. IMDB also contains 3 types of nodes and the learning goal is to tell the different genres of movies. In the comparison, we refer to the classification results of GCN [17], GAT [19], HAN [52], and GTN [53] which were presented in the material of GTN [53]. And we keep all the node embedding dimension to be 64 for all the methods including PINE. To keep the fairness of the comparison to the model size, we choose the same layer number settings as what used in GTN [53]. For the IMDB, we apply three PINE functions and for the ACM, we use two. We provide the comparison results in Table 4 . Since we follow the exact training, validation, and test splitting stated in GTN [53], we can directly compete the performance of PINE with other methods. Clearly, GTN is still the best performer for all datasets, and all graph neural network methods outperform the baseline methods based on the random walks. Although in these two datasets, PINE is not the best one (it is the second best one), we only modify the graph neural network layers into the PINE's universal partial permutation invariant function without specific design such as metapath generation mentioned in HAN or GTN. Therefore, with the universal approximation of PINE, we can easily obtain the compatible node classification performance on the heterogeneous graphs even compared with the method with specific graph neural network design.

\section{Conclusion ANd Future Work}

To summarize the whole paper, we propose PINE, a general graph embedding solution with the novel notion of partial permutation invariant set function, that in principle can capture arbitrary dependence among neighbors and automatically decide the significance of neighbor nodes at different distance for both homogeneous and heterogeneous graphs. We provide a theoretical guarantee for the effectiveness of the whole model. Through extensive experimental evaluation, we show that PINE offers better performance on both homogeneous and heterogeneous graphs, compared to stochastic trajectories based, matrix analytics based and graph neural network based state-of-the-art algorithms. For the future work, Our model can also be extended to more general cases, e.g., involving the rich content information out of graph neighborhood structures.

\section{REFERENCES}

[1] P. Goyal and E. Ferrara, "Graph embedding techniques, applications, and performance: A survey," Knowledge-Based Systems, vol. 151, pp. 78-94, 2018.

[2] H. Cai, V. W. Zheng, and K. C. Chang, "A comprehensive survey of graph embedding: Problems, techniques and applications," CoRR, vol. abs/1709.07604, 2017.

[3] S. Bhagat, G. Cormode, and S. Muthukrishnan, "Node classification in social networks," in Social network data analytics. Springer, 2011, pp. $115-148$.

[4] D. Liben-Nowell and J. Kleinberg, "The link-prediction problem for social networks," journal of the Association for Information Science and Technology, vol. 58, no. 7, pp. 1019-1031, 2007.

[5] S. Fortunato, "Community detection in graphs," Physics reports, vol. 486, no. 3-5, pp. 75-174, 2010.

[6] X. Yu, X. Ren, Y. Sun, Q. Gu, B. Sturt, U. Khandelwal, B. Norick, and J. Han, "Personalized entity recommendation: A heterogeneous information network approach," in WSDM, 2014, pp. 283-292.

[7] W. Hamilton, R. Ying, and J. Leskovec, "Representation learning on graphs: Methods and applications," CoRR, vol. abs/1709.05584, 2017.

[8] B. Perozzi, R. Al-Rfou, and S. Skiena, "Deepwalk: Online learning of social representations," in KDD, 2014, pp. 701-710.

[9] A. Grover and J. Leskovec, "node2vec: Scalable feature learning for networks," in KDD, 2016, pp. 855-864.

[10] Y. Dong, N. V. Chawla, and A. Swami, "metapath2vec: Scalable representation learning for heterogeneous networks," in KDD, 2017, pp. 135-144.

[11] G. H. Nguyen, J. B. Lee, R. A. Rossi, N. K. Ahmed, E. Koh, and S. Kim, "Continuous-time dynamic network embeddings," in Companion Proceedings of the The Web Conference 2018, 2018, pp. 969-976.

[12] Z. Zhang, P. Cui, X. Wang, J. Pei, X. Yao, and W. Zhu, "Arbitraryorder proximity preserved network embedding," in KDD, 2018, pp. 2778-2786.

[13] D. Wang, P. Cui, and W. Zhu, "Structural deep network embedding," in KDD, 2016, pp. 1225-1234.

[14] S. Cao, W. Lu, and Q. Xu, "Deep neural networks for learning graph representations," in AAAI, 2016, pp. 1145-1152.

[15] A. Bojchevski and S. Günnemann, "Deep gaussian embedding of graphs: Unsupervised inductive learning via ranking," in ICLR, 2018.

[16] Y. Chen, J. Pu, X. Liu, and X. Zhang, "Gaussian mixture embedding of multiple node roles in networks," World Wide Web, vol. 23, no. 2, pp. 927-950, 2020.

[17] T. N. Kipf and M. Welling, "Semi-supervised classification with graph convolutional networks," arXiv preprint arXiv:1609.02907, 2016.

[18] W. Hamilton, R. Ying, and J. Leskovec, "Inductive representation learning on large graphs," in NIPS '17, 2017, pp. 1024-1034.

[19] P. Velickovic, G. Cucurull, A. Casanova, A. Romero, P. Lio, and Y. Bengio, "Graph attention networks," arXiv preprint arXiv:1710.10903, vol. 1, no. 2, 2017. 
[20] K. K. Thekumparampil, C. Wang, S. Oh, and L.-J. Li, “Attentionbased graph neural network for semi-supervised learning," arXiv preprint arXiv:1803.03735, 2018.

[21] U. Akujuobi, Q. Zhang, H. Yufei, and X. Zhang, "Recurrent attention walk for semi-supervised classification," in WSDM, 2020, pp. $16-24$.

[22] U. Akujuobi, Y. Han, Q. Zhang, and X. Zhang, "Collaborative graph walk for semi-supervised multi-label node classification," in ICDM, 2019, pp. 1-10.

[23] F. Wu, A. H. S. Jr., T. Zhang, C. Fifty, T. Yu, and K. Q. Weinberger, "Simplifying graph convolutional networks," in ICML 2019, 2019, pp. 6861-6871.

[24] J. Tang, M. Qu, M. Wang, M. Zhang, J. Yan, and Q. Mei, "Line: Large-scale information network embedding," in Proceedings of the 24th International Conference on World Wide Web, 2015, pp. 1067-1077.

[25] C. Yang, M. Sun, Z. Liu, and C. Tu, "Fast network embedding enhancement via high order proximity approximation," in IJCAI, 2017, pp. 19-25.

[26] D. Zhu, P. Cui, D. Wang, and W. Zhu, “Deep variational network embedding in wasserstein space," in KDD, 2018, pp. 2827-2836.

[27] P. Goyal and E. Ferrara, "Graph embedding techniques, applications, and performance: A survey," CoRR, vol. abs/1705.02801, 2017.

[28] L. Tang and H. Liu, "Leveraging social media networks for classification," Data Mining and Knowledge Discovery, vol. 23, no. 3, pp. 447-478, 2011.

[29] S. Cao, W. Lu, and Q. Xu, "GraRep: Learning graph representations with global structural information," in CIKM, 2015, pp. 891-900.

[30] M. Ou, P. Cui, J. Pei, Z. Zhang, and W. Zhu, "Asymmetric transitivity preserving graph embedding," in $K D D$ '16. New York, NY, USA: ACM, 2016, pp. 1105-1114.

[31] R. A. Rossi, N. K. Ahmed, and E. Koh, "Higher-order network representation learning," in Companion of the The Web Conference 2018 on The Web Conference 2018, 2018, pp. 3-4.

[32] C. Yang, Z. Liu, D. Zhao, M. Sun, and E. Y. Chang, "Network representation learning with rich text information." in IJCAI, 2015, pp. 2111-2117.

[33] C. Tu, W. Zhang, Z. Liu, and M. Sun, "Max-margin deepwalk: Discriminative learning of network representation." in IJCAI, 2016 , pp. 3889-3895.

[34] R. Liao, Z. Zhao, R. Urtasun, and R. S. Zemel, "Lanczosnet: Multi-scale deep graph convolutional networks," arXiv preprint arXiv:1901.01484, 2019.

[35] X. Huang, J. Li, and X. Hu, "Label informed attributed network embedding," in WSDM, 2017, pp. 731-739.

[36] L. Lovász, "Random walks on graphs," Combinatorics, Paul erdos is eighty, vol. 2, no. 1-46, p. 4, 1993.

[37] T. Mikolov, I. Sutskever, K. Chen, G. S. Corrado, and J. Dean, "Distributed representations of words and phrases and their compositionality," in NIPS, 2013, pp. 3111-3119.

[38] L. F. Ribeiro, P. H. Saverese, and D. R. Figueiredo, "struc2vec Learning node representations from structural identity," in $K D D$, 2017, pp. 385-394.

[39] H. Gao and H. Huang, "Self-paced network embedding," in KDD '18. New York, NY, USA: ACM, 2018, pp. 1406-1415.

[40] Z. Yang, W. W. Cohen, and R. Salakhutdinov, "Revisiting semisupervised learning with graph embeddings," in $I C M L, 2016$, pp $40-48$.

[41] S. Chang, W. Han, J. Tang, G.-J. Qi, C. C. Aggarwal, and T. S. Huang, "Heterogeneous network embedding via deep architectures," in $K D D, 2015$, pp. 119-128.

[42] X. Wang, Y. Zhang, and C. Shi, "Hyperbolic heterogeneous information network embedding," in AAAI, 2019, pp. 5337-5344.

[43] J. Tang, M. Qu, and Q. Mei, "PTE: Predictive text embedding through large-scale heterogeneous text networks," in SIGKDD, 2015, pp. 1165-1174.

[44] C. Donnat, M. Zitnik, D. Hallac, and J. Leskovec, "Learning structural node embeddings via diffusion wavelets," in SIGKDD, 2018, pp. 1320-1329.

[45] T. N. Kipf and M. Welling, "Variational graph auto-encoders," arXiv preprint arXiv:1611.07308, 2016.

[46] M. Schlichtkrull, T. N. Kipf, P. Bloem, R. van den Berg, I. Titov, and M. Welling, "Modeling relational data with graph convolutional networks," in European Semantic Web Conference, 2018, pp. 593-607.

[47] R. van den Berg, T. N. Kipf, and M. Welling, "Graph convolutional matrix completion," stat, vol. 1050, p. 7, 2017.
[48] J. Chen, T. Ma, and C. Xiao, "FastGCN: Fast learning with graph convolutional networks via importance sampling," in ICLR, 2018.

[49] T. Pham, T. Tran, D. Q. Phung, and S. Venkatesh, "Column networks for collective classification." in AAAI, 2017, pp. 2485-2491.

[50] K. Tu, P. Cui, X. Wang, P. S. Yu, and W. Zhu, "Deep recursive network embedding with regular equivalence," in $K D D, 2018$, pp. 2357-2366.

[51] K. Xu, W. Hu, J. Leskovec, and S. Jegelka, "How powerful are graph neural networks?" arXiv preprint arXiv:1810.00826, 2018

[52] X. Wang, H. Ji, C. Shi, B. Wang, Y. Ye, P. Cui, and P. S. Yu, "Heterogeneous graph attention network," in The World Wide Web Conference, 2019, pp. 2022-2032.

[53] S. Yun, M. Jeong, R. Kim, J. Kang, and H. J. Kim, “Graph transformer networks," in NeurIPS, 2019, pp. 11983-11993.

[54] Y. Lu, C. Shi, L. Hu, and Z. Liu, "Relation structure-aware heterogeneous information network embedding," in $A A A I$, vol. 33, 2019, pp. 4456-4463.

[55] H. Hong, H. Guo, Y. Lin, X. Yang, Z. Li, and J. Ye, "An attentionbased graph neural network for heterogeneous structural learning." in AAAI, 2020, pp. 4132-4139.

[56] A. Loukas, "What graph neural networks cannot learn: depth vs width," arXiv preprint arXiv:1907.03199, 2019.

[57] J. Gilmer, S. S. Schoenholz, P. F. Riley, O. Vinyals, and G. E. Dahl, "Neural message passing for quantum chemistry," arXiv preprint arXiv:1704.01212, 2017.

[58] M. Zaheer, S. Kottur, S. Ravanbakhsh, B. Poczos, R. R. Salakhutdinov, and A. J. Smola, "Deep sets," in NIPS, 2017, pp. 3394-3404.

[59] D. Yarotsky, "Universal approximations of invariant maps by neural networks," arXiv preprint arXiv:1804.10306, 2018.

[60] G. Cybenko, "Approximations by superpositions of a sigmoidal function," Mathematics of Control, Signals and Systems, vol. 2, pp. 183-192, 1989.

[61] K. Hornik, "Approximation capabilities of multilayer feedforward networks," Neural networks, vol. 4, no. 2, pp. 251-257, 1991.

[62] A. K. McCallum, K. Nigam, J. Rennie, and K. Seymore, "Automating the construction of internet portals with machine learning," Information Retrieval, vol. 3, no. 2, pp. 127-163, 2000.

[63] C. L. Giles, K. D. Bollacker, and S. Lawrence, "Citeseer: An automatic citation indexing system," in Proceedings of the third ACM conference on Digital libraries. ACM, 1998, pp. 89-98.

[64] P. Sen, G. Namata, M. Bilgic, L. Getoor, B. Galligher, and T. Eliassi$\mathrm{Rad}$, "Collective classification in network data," AI magazine, vol. 29, no. 3, p. 93, 2008.

[65] J. Leskovec, J. Kleinberg, and C. Faloutsos, "Graph evolution: Densification and shrinking diameters," ACM Transactions on Knowledge Discovery from Data (TKDD), vol. 1, no. 1, p. 2, 2007.

[66] S. Abu-El-Haija, B. Perozzi, R. Al-Rfou, and A. Alemi, "Watch your step: Learning node embeddings via graph attention," in NeurIPS, 2018.

[67] M. Ji, Y. Sun, M. Danilevsky, J. Han, and J. Gao, "Graph regularized transductive classification on heterogeneous information networks," in Joint European Conference on Machine Learning and Knowledge Discovery in Databases. Springer, 2010, pp. 570-586.

[68] X. Wang, L. Tang, H. Gao, and H. Liu, "Discovering overlapping groups in social media," in ICDM2010, 2010.

[69] H. Weyl, The classical groups: their invariants and representations. Princeton university press, 1946.

[70] M. H. Stone, "Applications of the theory of boolean rings to general topology," Transactions of the American Mathematical Society, vol. 41, no. 3, pp. 375-481, 1937.

[71] — - "The generalized weierstrass approximation theorem," Mathematics Magazine, vol. 21, no. 5, pp. 237-254, 1948.

[72] H. Kraft and C. Procesi, Classical Invariant Theory, a Primer. Lecture notes, 2000

[73] G. Cybenko, "Approximation by superpositions of a sigmoidal function," Mathematics of control, signals and systems, vol. 2, no. 4, pp. 303-314, 1989.

[74] T. Derr, Y. Ma, and J. Tang, "Signed graph convolutional networks," in ICDM, 2018, pp. 929-934.

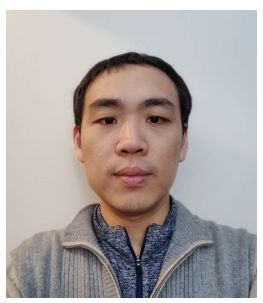

Shupeng Gui received the B.E. degree in Electrical engineering and information science from University of Science and Technology of China, the M.S. degree in Computer Science from University of Rochester in 2015, and 2018, respectively. $\mathrm{He}$ is a $\mathrm{PhD}$ Candidate in Department of Computer Science in University of Rochester. His research focuses on Graph Embedding, Adversarial Learning, and DNN Model Compression. 


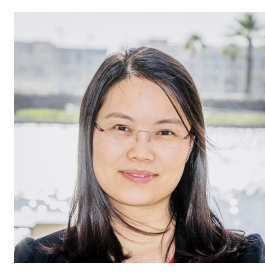

Xiangliang Zhang is currently an Associate Professor and directs the Machine Intelligence and Knowledge Engineering (MINE) Laboratory at the Division of Computer, Electrical, and Mathematical Sciences and Engineering, King Abdullah University of Science and Technology (KAUST) Saudi Arabia. She received the Ph.D. degree in computer science from INRIA-University ParisSud, France, in July 2010. Her current research interests and experiences include machine learning, and data mining. She was invited to deliver an Early Career Spotlight talk at IJCAI-ECAI 2018.

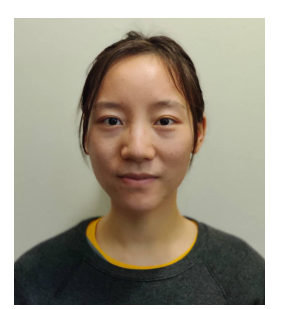

Pan Zhong is a PhD student of Department of Electrical and Computer Engineering in lowa State University. She received her B.E. degree in Electrical Engineering and Information Science from University of Science and Technology of China in 2015. Her research focuses on invariant and equivaraint deep neural networks.

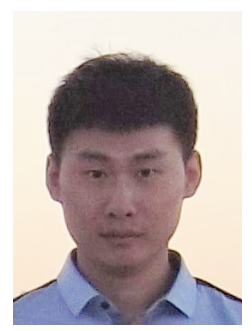

Shuang Qiu is working toward the $\mathrm{PhD}$ degree in computer science and engineering at the University of Michigan. His research interests include machine learning theory and its application to data mining.

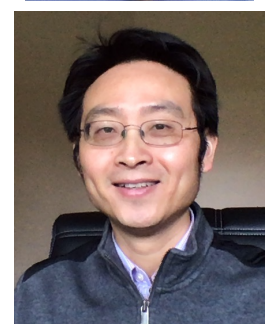

Mingrui Wu received the bachelor's degree and the Ph.D. degree in the Compute Science and Technology department from Tsinghua University China, in 1996 and 2001, respectively. He is currently a Software Engineer and Tech Lead in Facebook, working on Ad Ranking. Prior to joining Facebook, he worked as Senior Director of Engineering in PlusAl, working on self-driving trucks. Before he had worked as Senior Staff Engineer and Director in Alibaba, and Principal Development Lead in Microsoft. His research and working interests include machine learning, personalized search and recommendation, Ad ranking, and autonomous driving.

Jieping Ye received his Ph.D. degree in computer science from the University of Minnesota, Twin Cities, MN, USA, in 2005. He is a VP of Beike. He is also a Professor with the University of Michigan, Ann Arbor, MI, USA. His research interests include big data, machine learning, and data mining with applications in transportation and biomedicine. He won the NSF CAREER Award in 2010 and the 2019 Daniel H. Wagner Prize for Excellence in the Practice of Advanced Analytics and Operations. His papers have been selected for the Outstanding Student Paper at ICML in 2004, the KDD Best Research Paper Runner Up in 2013, and the KDD Best Student Paper Award in 2014. He has served as a Senior Program Committee/Area Chair/Program Committee Vice Chair of many conferences, including NIPS, ICML, KDD, IJCAI, ICDM, and SDM. He has served as an Associate Editor for Data Mining and Knowledge Discovery and IEEE Transactions on Pattern Analysis and Machine Intelligence.

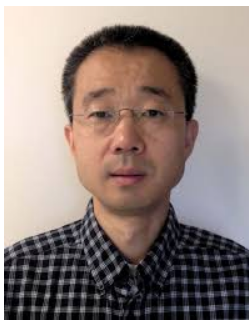

Zhengdao Wang Zhengdao Wang received his Bachelor's degree in Electronic Engineering and Information Science from the University of Science and Technology of China (USTC), 1996, the M.Sc. degree in Electrical and Computer Engineering from the University of Virginia, 1999, and Ph.D. in Electrical and Computer Engineering from the University of Minnesota, 2002. He is now with the Department of Electrical and Computer Engineering at the lowa State University. His interests are in the areas of signal processing communications, and information theory, and machine learning. He was a co-recipient of the IEEE Signal Processing Magazine Best Paper Award in 2003 and the IEEE Communications Society Marconi Paper Prize Award in 2004, and the EURASIP Journal on Advances in Signal Processing Best Paper Award, in 2009.

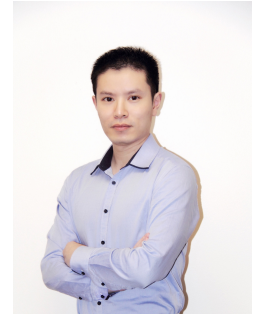

Ji Liu Dr. Ji Liu is currently the head of $\mathrm{Al}$ platform department and Seattle Al lab at Kwai Inc. $\mathrm{He}$ is also an affiliated professor at University of Rochester. He graduated from University of Wisconsin-Madison in computer science. He worked in machine learning and artificial intelligence for more than 15 years. His research covers a wide range of $\mathrm{ML}$ and $\mathrm{Al}$ areas, such as optimization, computer vision, reinforcement learning, recommendation system, bioinformatics, robotics, etc. He received the IBM faculty award in 2017 and a few best paper or nomination awards in top tier CS conferences such as UAI and KDD. He is also an awardee of MIT TR35 China 2017. He serves the ML community as an AC, SPC, or PC for top ML conferences such as AAAI, IJCAI, NIPS, ICML, etc. 


\section{APPENDIX A}

\section{PARTIAL PERMUTATION INVARIANT MAPS}

\section{A.1 Definitions: Permutation Invariant Maps and Poly- nomials}

Definition A.1. [Symmetric group $S_{N}$ ] Given an index set $\mathbb{A}=\{1,2, \cdots, N\}$, the set of all one-to-one mappings $\pi: \mathbb{A} \longrightarrow$ $\mathbb{A}$ forms the symmetric group $S_{N}$ with function compositions as the group action.

For brevity, we will denote $\boldsymbol{X}:=\left[\mathrm{x}_{1}, \mathbf{x}_{2}, \cdots, \mathbf{x}_{N}\right]$. And its permutation is denoted as

$$
T_{\pi} \boldsymbol{X}:=\left[\mathbf{x}_{\pi(1)}, \mathbf{x}_{\pi(2)}, \cdots, \mathbf{x}_{\pi(N)}\right]
$$

for an arbitrary $\boldsymbol{X} \in \mathbb{R}^{M \times N}$, where $\pi \in S_{N}, \pi(n) \in \mathbb{A}$ is the index at position $n$ after permutation and $\mathbf{x}_{\pi(n)}$ is the $\pi(n)$-th column of $\boldsymbol{X}$. The $T_{\pi} \boldsymbol{X}$ defined here is equivalent to the permutation matrix notation we used in Definition 3.1. As the proofs are based on symmetric group action, we also give the definition of permutation invariant map and partially permutation invariant map based on symmetric group action in the following.

Definition A.2. [Permutation invariant map or $S_{N^{-}}$invariant map] $A$ continuous real valued map $f: \mathbb{R}^{M \times N} \longrightarrow \mathbb{R}$ is permutation invariant if

$$
f\left(T_{\pi} \boldsymbol{X}\right)=f(\boldsymbol{X})
$$

for all $\pi \in S_{N}$ and all $\boldsymbol{X} \in \mathbb{R}^{M \times N}$.

Definition A.3. [Partially permutation invariant map] Given a series of symmetric group $S_{N_{1}}, S_{N_{2}}, \cdots, S_{N_{K}}$, and $\mathbb{W}:=\mathbb{W}_{1} \times \mathbb{W}_{2} \cdots \times \mathbb{W}_{K}$, where $\mathbb{W}_{k}$ is an arbitrary matrix space, a continuous real valued map $f: \mathbb{W} \longrightarrow \mathbb{R}$ is partially permutation invariant if

$$
f\left(T_{\pi_{1}} \boldsymbol{X}_{1}, T_{\pi_{2}} \boldsymbol{X}_{2}, \cdots, T_{\pi_{K}} \boldsymbol{X}_{K}\right)=f\left(\boldsymbol{X}_{1}, \boldsymbol{X}_{2}, \cdots, \boldsymbol{X}_{K}\right)
$$

for all $\pi_{k} \in S_{N_{k}}$ and all $\boldsymbol{X}_{k} \in \mathbb{W}_{k}$.

We call a polynomial that is a permutation invariant map permutation invariant polynomial. When the symmetric group involved is significant, we will use terms like $S_{N^{-}}$ invariant polynomial. Similarly, a polynomial that is a partially permutation invariant map will be called partially permutation invariant polynomial.

\section{A.2 Proof of Theorem 3.2}

A sketch of the proof ideas is presented as follows.

1) We first show that partially permutation invariant polynomials are dense in partially invariant continuous functional space in Lemma A.5 based on that StoneWeierstrass theorem states that polynomials are dense in the space of continuous functions.

2) Next, our proof goal is to find a finite generating set for the partially invariant functions so that any partially invariant function can be represented as a polynomial of that generating set.

There is a well-known generating set for permutation invariant polynomials $f: \mathbb{R}^{N} \longrightarrow \mathbb{R}$ : the power sum polynomials. Polarization is introduced to extend the generating set to the case of $f: \mathbb{R}^{M \times N} \longrightarrow \mathbb{R}$.

\section{A.2.1 Polarization}

The goal of polarization is to represent polynomial invariant of a higher dimension in terms of invariant polynomials of a lower dimension. The lemma in the following provides an invariant representation of matrix-argument invariant polynomial in terms of vector-argument invariant polynomial.

The group $S_{N}$ only permutes the columns of $\mathbb{R}^{M \times N}$. So given two spaces $\mathbb{W}:=\mathbb{R}^{M \times N}$ and $\mathbb{W}^{\prime}:=\mathbb{R}^{M^{\prime} \times N}$, and a linear mapping

$$
\mathbf{A}: \mathbb{W} \longrightarrow \mathbb{W}^{\prime}
$$

$\mathbf{A}$ is commutable with permutation; i.e., $\mathbf{A}\left(T_{\pi} \boldsymbol{X}\right)=$ $T_{\pi}(\mathbf{A}(\boldsymbol{X}))=T_{\pi}(\boldsymbol{A} \boldsymbol{X})$, where $\mathbf{A}(\boldsymbol{X})=\boldsymbol{A} \boldsymbol{X}$. If $f$ is a permutation invariant on $\mathbb{W}^{\prime}$ then $f \circ \mathbf{A}$ is permutation invariant on $\mathbb{W}$. In the following lemma, we take $M^{\prime}=1$.

Lemma A.4. [Weyl's Polarization] For any polynomial invariant $f$ on $\mathbb{R}^{M \times N}$, there exist a series of $1 \times M$ vectors $\left\{\boldsymbol{a}_{t}\right\}_{t=1}^{T}$ and a series of $S_{N}$-invariant polynomials $\rho_{t}$ on $\mathbb{R}^{1 \times N}$, such that $f$ can be represented by

$$
f(\boldsymbol{X})=\sum_{t=1}^{T} \rho_{t}\left(\boldsymbol{a}_{t}^{\top} \boldsymbol{X}\right)
$$

Proof. This result is form of Weyl's poloarization [69]. It follows, e.g., from the Theorem 2.3 in $[59]$ by taking the multiplicities $m_{\alpha}^{\prime}$ of the $\Gamma$-modules $V_{\alpha}^{\prime}$ equal to 1 , taking the group to be $S_{N}$, and taking the $\rho_{t}$ 's from the generating set of vector-argument invariant polynomials.

\section{A.2.2 Partially Permutation Invariant Polynomials}

The following lemma shows that it is sufficient to use partially invariant polynomials to approximate partially invariant functions.

Lemma A.5. [Denseness of Partially Invariant Polynomials] For any partially invariant function $f$ on $\mathbb{W}:=$ $\mathbb{W}_{1} \times \mathbb{W}_{2} \cdots \times \mathbb{W}_{K}$ where $\mathbb{W}_{k}:=\mathbb{R}^{M_{k} \times N_{k}}$ and for any $\epsilon>0$, there exists a partially invariant polynomial $\hat{f}$ such that $|\hat{f}(\mathcal{X})-f(\mathcal{X})|<\epsilon$ for all $\mathcal{X} \in \mathbb{W}$.

Proof. By Stone-Weierstrass Theorem [70], [71] for the compact Hausdorff space, polynomial on compact Hausdorff space is dense in the space of continuous functions on that same compact Hausdorff space. So for any partially invariant function $f$ on $\mathbb{W}$ and any $\epsilon>0$, there exists a polynomial $f^{\prime}$ on $\mathbb{W}$ such that $\left|f^{\prime}(\mathcal{X})-f(\mathcal{X})\right|<\epsilon$ for all $\mathcal{X} \in \mathbb{W}$.

Let $\mathcal{X}=\left\{\boldsymbol{X}_{1}, \cdots, \boldsymbol{X}_{K}\right\} \in \mathbb{W}$, and $|\mathcal{S}|=\left|S_{N_{1}}\right| \cdots\left|S_{N_{K}}\right|$. We can construct

$$
\hat{f}(\mathcal{X})=\frac{1}{|\mathcal{S}|} \sum_{\substack{\pi_{1} \in S_{N_{1}} \ldots \\ \pi_{K} \in S_{N_{K}}}} f^{\prime}\left(T_{\pi_{1}} \boldsymbol{X}_{1}, \cdots, T_{\pi_{K}} \boldsymbol{X}_{K}\right)
$$


which is a partially invariant polynomial. We have

$$
\begin{aligned}
& |\hat{f}(\mathcal{X})-f(\mathcal{X})| \\
& =\left|\frac{1}{|\mathcal{S}|} \sum_{\substack{\pi_{1} \in S_{N_{1}} \ldots \\
\pi_{K} \in S_{N_{K}}}} f^{\prime}\left(T_{\pi_{1}} \boldsymbol{X}_{1}, \cdots, T_{\pi_{K}} \boldsymbol{X}_{K}\right)-f(\mathcal{X})\right| \\
& =\mid \frac{1}{|\mathcal{S}|} \sum_{\substack{\pi_{1} \in S_{N_{1}} \ldots \\
\pi_{K} \in S_{N_{K}}}}\left(f^{\prime}\left(T_{\pi_{1}} \boldsymbol{X}_{1}, \cdots, T_{\pi_{K}} \boldsymbol{X}_{K}\right)\right. \\
& \leq \frac{1}{|\mathcal{S}|} \sum_{\substack{\pi_{1} \in S_{N_{1}} \cdots \\
\pi_{K} \in S_{N_{K}}}} \mid f^{\prime}\left(T_{\pi_{1}} \boldsymbol{X}_{1}, \cdots, T_{\pi_{K}} \boldsymbol{X}_{K}\right) \\
& <\epsilon .
\end{aligned}
$$

The function in (11) thus fulfills the requirement of the lemma.

The following lemma gives one form of explicit expansion for partially invariant polynomials.

Lemma A.6. Any partially invariant polynomial $f$ on $\mathbb{W}:=$ $\mathbb{W}_{1} \times \mathbb{W}_{2} \cdots \times \mathbb{W}_{K}$, where $\mathbb{W}_{k}:=\mathbb{R}^{M_{k} \times N_{k}}$, can be expressed in the following form:

$$
f(\mathcal{X})=\sum_{q=1}^{Q} g_{1, q}\left(\boldsymbol{X}_{1}\right) g_{2, q}\left(\boldsymbol{X}_{2}\right) \cdots g_{K, q}\left(\boldsymbol{X}_{K}\right),
$$

where $Q$ is an integer, and $g_{k, q}\left(\boldsymbol{X}_{k}\right)$ are $S_{N_{k}}$-invariant.

Proof. Since $f$ is a polynomial, the function $f$ can be expressed as

$$
f(\mathcal{X})=\sum_{q=1}^{Q} g_{1, q}^{\prime}\left(\boldsymbol{X}_{1}\right) g_{2, q}^{\prime}\left(\boldsymbol{X}_{2}\right) \cdots g_{K, q}^{\prime}\left(\boldsymbol{X}_{K}\right),
$$

where $Q$ is a suitable integer depending on the degree of $f$, and $g_{k, q}^{\prime}$ are polynomials on $\mathbb{W}_{k}$.

Define the symmetrized versions of $g_{k, q}^{\prime}$ as follows:

$$
g_{k, q}\left(\boldsymbol{X}_{k}\right):=\frac{1}{\left|S_{N_{k}}\right|} \sum_{\pi_{k} \in S_{N_{k}}} g_{k, q}^{\prime}\left(T_{\pi_{k}} \boldsymbol{X}_{k}\right) .
$$

As $f$ is partially invariant, its value does not change if we perform (partial) symmetrization on $f$. Therefore,

$$
\begin{aligned}
& f(\mathcal{X})=\frac{1}{|\mathcal{S}|} \sum_{\substack{\pi_{1} \in S_{N_{1}} \cdots \\
\pi_{K} \in S_{N_{K}}}} f\left(T_{\pi_{1}} \boldsymbol{X}_{1}, \cdots, T_{\pi_{K}} \boldsymbol{X}_{K}\right) \\
& =\frac{1}{|\mathcal{S}|} \sum_{\substack{\pi_{1} \in S_{N_{1}} \ldots \\
\pi_{K} \in S_{N_{K}}}} \sum_{q=1}^{Q} g_{1, q}^{\prime}\left(T_{\pi_{1}} \boldsymbol{X}_{1}\right) \cdots g_{K, q}^{\prime}\left(T_{\pi_{K}} \boldsymbol{X}_{K}\right) \\
& =\sum_{q=1}^{Q} g_{1, q}\left(\boldsymbol{X}_{1}\right) \cdots g_{K, q}\left(\boldsymbol{X}_{K}\right),
\end{aligned}
$$

where the last step follows by exchanging the order of summations, and distributing the symmetrization sums to their respective $g_{k, q}^{\prime}$ functions.
Lemma A.7. [Hilbert's finiteness Theorem, e.g., [72]] There exists finitely many invariant polynomials $f_{1}, \ldots, f_{N_{\text {inv }}}: \mathbb{R}^{n} \rightarrow$ $\mathbb{R}$ such that any invariant polynomial $f: \mathbb{R}^{n} \rightarrow \mathbb{R}$ can be expressed as

$$
f(\mathbf{x})=\tilde{f}\left(f_{1}(\mathbf{x}), \cdots, f_{N_{\text {inv }}}(\mathbf{x})\right)
$$

with some polynomial $\tilde{f}$ of $N_{\text {inv }}$ variables.

Lemma A.8. [Power sums as generating set] One generating set of symmetric polynomials on $\mathbb{R}^{N}$ is power sums up to degree $N$ :

$$
f_{j}(\mathbf{x})=\sum_{n=1}^{N} x_{n}^{j}, \quad j=1, \cdots, N,
$$

where $x_{n}$ is the $n$-th entry of $\mathbf{x}$.

\section{A.2.3 Proof of Theorem 3.2}

Here comes to the core of the proof which supports the Representation Theorem of Partial Permutation Invariant Set Functions stated in Theorem 3.2 Let's recall the goal which is to figure out the universal approximation formulation for an arbitrary partial permutation invariant set function f. The sketch of the proof is that we decompose the partial permutation invariant function into polynomials as the first step and then show these polynomials can be composed into a specific form presented in Theorem 3.2 .

Proof. By Lemma A.5. any partially invariant function can be approximated by a partially invariant polynomial, which in turn can be written in the form in the equation (12), due to Lemma A.6 Using Lemma A.4, each term (fully) invariant polynomial $g_{k, q}\left(\boldsymbol{X}_{k}\right)$ can be expressed as follows,

$$
g(\boldsymbol{X})=\sum_{t=1}^{T} \rho_{t}\left(\boldsymbol{a}_{t}^{\top} \boldsymbol{X}\right),
$$

where $\rho_{t}$ is an invariant polynomial, and we ignore the notation with $(k, q)$ for simplicity.

Based on Hilbert's finiteness Theorem in Lemma A.7, and the power-sum basis result in Lemma A.8. each function $\rho_{t}\left(\boldsymbol{a}_{t}^{\top} \boldsymbol{X}\right)$ is expressible as a polynomial of the following $N \cdot T$ variables:

$$
\left\{\sum_{n=1}^{N}\left(\boldsymbol{a}_{t}^{\top} \mathbf{x}_{n}\right)^{j} \mid \quad t \in[T], \quad j \in[N]\right\},
$$

where $\mathbf{x}_{n}$ is the $n$-th column in $\boldsymbol{X}$.

Let's collect these $\left\{\boldsymbol{a}_{t} \mid t \in[T]\right\}$ in a matrix $\boldsymbol{A} \in \mathbb{R}^{M \times T}$ whose $t$-th column is $\boldsymbol{a}_{t}$. We define the power-sum vector function $\mathbf{p}^{(N)}: \mathbb{R} \rightarrow \mathbb{R}^{N}$ :

$$
\mathbf{p}^{(N)}(x):=\left[x^{1}, x^{2}, \ldots, x^{N}\right],
$$

and we define the function $\mathrm{g}: \mathbb{R}^{M} \rightarrow \mathbb{R}^{N \cdot T}$ :

$$
\mathbf{g}(\mathbf{x} ; \boldsymbol{A}):=\left[\mathbf{p}^{(N)}\left(\boldsymbol{a}_{1}^{\top} \mathbf{x}\right), \mathbf{p}^{(N)}\left(\boldsymbol{a}_{2}^{\top} \mathbf{x}\right), \ldots, \mathbf{p}^{(N)}\left(\boldsymbol{a}_{T}^{\top} \mathbf{x}\right)\right] .
$$

Therefore, $\left[\rho_{1}\left(\boldsymbol{a}_{1}^{\top} \boldsymbol{X}\right), \ldots, \rho_{T}\left(\boldsymbol{a}_{T}^{\top} \boldsymbol{X}\right)\right]$ can be expressed as a polynomial of

$$
\sum_{n=1}^{N} \mathbf{g}\left(\mathbf{x}_{n} ; \boldsymbol{A}\right) \text {. }
$$

Hence, the function $g(\boldsymbol{X})$ can also be compressed as a polynomial of (17). And for an arbitrary $(k, q)$ pair, we have 
the expression (17) to be $\sum_{n=1}^{N} \mathbf{g}_{k, q}\left(\mathbf{x}_{k, n} ; \boldsymbol{A}_{k, q}\right)$. For simplicity, we define $\mathcal{A}_{k}:=\left\{\boldsymbol{A}_{k, 1}, \ldots, \boldsymbol{A}_{k, Q}\right\}$, and the function $\mathbf{g}\left(\mathbf{x} ; \mathcal{A}_{k}\right):=\left[\mathbf{g}_{k, 1}\left(\mathbf{x} ; \boldsymbol{A}_{k, 1}\right), \ldots, \mathbf{g}_{k, Q}\left(\mathbf{x} ; \boldsymbol{A}_{k, Q}\right)\right]$. By recalling Lemma A.6. we can establish that a parital permutation invariant function $f$ can be approximated arbitrarily well by a function of the form

$$
h\left(\sum_{n=1}^{N_{1}} \mathbf{g}_{1}\left(\mathbf{x}_{1, n} ; \mathcal{A}_{1}\right), \ldots, \sum_{n=1}^{N_{K}} \mathbf{g}_{K}\left(\mathbf{x}_{K, n} ; \mathcal{A}_{K}\right)\right),
$$

where $h$ is a properly designed polynomial. For simplicity, we show the form of $\mathrm{g}(\cdot)$ without the $\mathcal{A}$ in the statement of the theorem since a $\mathcal{A}$ is specific designed for a corresponding function $\mathbf{g}(\cdot)$.

\section{A.3 Partially Permutation Invariant Neural Network: Proof of Theorem 3.3}

The main idea of the proof is the following: as neural network is an universal approximator, we use neural networks with one hidden layer to approximate $h$ in (A.2.3 and the power functions. We then get an approximator of partially permutation invariant in the form of a structured neural network.

Proof. We see that A.2.3 can approximate any partially permutation invariant polynomial on $\mathbb{W}$. By the universal approximation theorem of neural networks [73], we can approximate the polynomial $h$ with a shallow (one-hidden layer) neural network. Any partially permutation invariant function can be approximated as

$$
\sum_{l=1}^{L} c_{l} \sigma\left(\sum_{k=1}^{K} \sum_{t=1}^{T_{k}} \sum_{j=1}^{N_{k}} w_{l, t j} \sum_{n=1}^{N_{k}} p_{j}\left(\boldsymbol{a}_{k, t}^{\top} \boldsymbol{x}_{k, n}\right)+b_{l}\right),
$$

where $p_{j}(x)=x^{j}$, and we have combined the double indices $q$ and $t$ of $\boldsymbol{a}_{k, q, t}$ in (16) into a single index $t$ for a fixed $k$.

It is clear that the function in (18) is partially permutation invariant as all the $\left\{\boldsymbol{x}_{k, n}\right\}_{n=1}^{N_{k}}$ are treated the same. We can approximate $p_{j}(y)$ by $\sum_{l^{\prime}=1}^{L^{\prime}} d_{j, l^{\prime}} \sigma\left(u_{j, l^{\prime}} y+v_{j, l^{\prime}}\right)$. Combining the two neural network approximators, it follows that the functions $h(\cdot)$ and $\boldsymbol{g}_{k}$ in Theorem 3.2 can be chosen in the following form:

$$
\begin{gathered}
\mathbf{h}\left(\left[\mathbf{z}_{1}^{\top}, \cdots, \mathbf{z}_{K}^{\top}\right]^{\top}=: \overline{\mathbf{z}} \mid \boldsymbol{c}, \boldsymbol{W}, \boldsymbol{b}\right)=\boldsymbol{c}^{\top} \boldsymbol{\sigma}(\boldsymbol{W} \overline{\mathbf{z}}+\boldsymbol{b}), \\
\mathbf{g}_{k}\left(\mathbf{x} \mid T, \boldsymbol{u},\left\{\boldsymbol{a}_{t}, \boldsymbol{b}_{t}^{\prime}\right\}_{t=1}^{T}\right)=\left[\begin{array}{c}
\boldsymbol{\sigma}\left(\left(\boldsymbol{u} \otimes \boldsymbol{a}_{1}\right) \mathbf{x}+\boldsymbol{b}_{1}^{\prime}\right) \\
\boldsymbol{\sigma}\left(\left(\boldsymbol{u} \otimes \boldsymbol{a}_{2}\right) \mathbf{x}+\boldsymbol{b}_{2}^{\prime}\right) \\
\vdots \\
\boldsymbol{\sigma}\left(\left(\boldsymbol{u} \otimes \boldsymbol{a}_{T}\right) \mathbf{x}+\boldsymbol{b}_{T}^{\prime}\right)
\end{array}\right],
\end{gathered}
$$

where we have omitted the index $k$ on $\boldsymbol{a}_{k, t}^{\top}$ and used $\boldsymbol{a}$ to denote $\boldsymbol{a}^{\top}$ for simplicity.

\section{APPENDIX B \\ Additional Experiment Setups}

\section{B.1 Configurations of Hardware and Software.}

PINE is implemented with PyTorch and Tensorflow learning framework in the version 1.1.0 and 1.8.0 (Python 3 version). All experiments are conducted on a Linux 18.04 machine. The machine has one Core i7-6700K, 64 GB RAM, 512GB+2T Hard disks and two GTX 1080 graphics cards.

\section{B.2 Train-Test Splits.}

For all homogeneous datasets, we conduct the same training and test splits for 5 times. In each round, we randomly sample a ratio of nodes from $10 \%$ to $90 \%$ to be the training set. We leave all the other nodes in the test set to evaluate the classification performance among all the methods. For the heterogeneous case, we only split the author nodes into training and test set since only author takes labels in those two datasets. We follow the same split strategy of homogeneous cases on the heterogeneous graphs as well.

\section{B.3 Case Study: PINE on link prediction task}

Furthermore, we propose another experiment to show PINE is also effective on signed link prediction. SGCN |74| considers a link prediction problem based on a signed edge graph setting. In the signed link prediction problem, we are supposed to predict the signs (positive or negative) of nonobserved edges with the knowledge of observed edges in a graph. As stated by [74], we concatenate the embedding vectors of two nodes in a graph to predict the sign of an edge between them. Therefore, such a problem is transformed into a binary classification on a set of pair of nodes. In the signed link prediction, we are required to predict whether positive or negative a non-observed edge is with other observed signed edges in the same graph. As stated by [74], we concatenate the embedding vectors of two nodes in a graph to predict the sign of an edge between them and compare the performance with SGCN [74]. The results in Table 5 show that PINE outperforms the baselines on both AUC and F1, except having similar AUC with SGCN on the dataset of Bitcoin-Alpha. 
TABLE 5: Link sign prediction results with AUC and F1 for Bitcoin-Alpha and Bitcoin-OTC datasets.

\begin{tabular}{cc|ccccc}
\hline & & SSE & SINE & SIDE & SGCN & PINE \\
\hline \multirow{2}{*}{ BItcoin-AlPHA } & AUC & 0.764 & 0.778 & 0.630 & $\mathbf{0 . 7 9 6}$ & 0.795 \\
& F1 & 0.898 & 0.888 & 0.738 & 0.917 & $\mathbf{0 . 9 5 4}$ \\
\hline \multirow{2}{*}{ BItcoIN-OTC } & AUC & 0.803 & 0.814 & 0.618 & 0.803 & $\mathbf{0 . 8 1 6}$ \\
& F1 & 0.923 & 0.878 & 0.750 & 0.925 & $\mathbf{0 . 9 2 7}$ \\
\hline
\end{tabular}




\section{Supplemental Material: How to extend P INE from 1-hop neighbors to k-hop neighbors}

In this section, we consider a problem how to consider the importance of neighbors at different distances (multi-hops). We next formulate our embedding model that can consider different order of neighborhood by characterizing $\mathbf{h}(\cdot)$ and $\left\{\mathbf{g}_{k}(\cdot)\right\}_{k=1}^{K}$.

1-step neighbors By Theorem 3.2. any mapping function of a node $\mathrm{v} \in \mathcal{V}_{k}$ can be characterized by appropriately designed functions $\mathbf{g}_{1}, \mathbf{g}_{2}, \cdots, \mathbf{g}_{K}$, and $\mathbf{h}_{k}$ :

$\mathbf{x}^{\mathrm{v}}=\mathbf{h}_{k}\left(\sum_{\mathrm{u} \in \Omega_{1,1}^{\mathrm{v}}} \mathbf{g}_{1}\left(\mathbf{x}^{\mathrm{u}}\right), \sum_{\mathrm{u} \in \Omega_{1,2}^{\mathrm{v}}} \mathbf{g}_{2}\left(\mathbf{x}^{\mathrm{u}}\right), \cdots, \sum_{\mathrm{u} \in \Omega_{1, K}^{\mathrm{v}}} \mathbf{g}_{K}\left(\mathbf{x}^{\mathrm{u}}\right)\right)$ $\forall \mathrm{v} \in \mathcal{V}_{k}, \forall k \in\{1, \ldots, K\}$,

where $\Omega_{n, k}^{\mathrm{v}}$ denotes the set of $n$-step neighbors of node $\mathrm{v}$ in node type $k$. Specifically, let $n=1$ for the case of 1-step neighbors.

Multi-step neighbors High order proximity has been shown to be beneficial to generating high quality embedding vectors [25]. Extending the 1-step neighbor model, we can have a more general model where the representation of each node could depend on 1-step (immediate) neighbors, 2-step neighbors, 3-step neighbors, and even infinite-step neighbors.

$$
\begin{gathered}
\mathbf{x}^{\mathrm{v}}=\mathbf{h}_{k}\left(\sum_{n=0}^{\infty} \alpha_{n} \sum_{\mathrm{u} \in \Omega_{n, 1}^{v}} \mathbf{g}_{1}\left(\mathbf{x}^{\mathrm{u}}\right), \sum_{n=0}^{\infty} \alpha_{n} \sum_{\mathbf{u} \in \Omega_{n, 2}^{\mathrm{v}}} \mathbf{g}_{2}\left(\mathbf{x}^{\mathrm{u}}\right), \cdots,\right. \\
\left.\sum_{n=0}^{\infty} \alpha_{n} \sum_{\mathrm{u} \in \Omega_{n, K}^{\mathrm{v}}} \mathbf{g}_{K}\left(\mathbf{x}^{\mathrm{u}}\right)\right), \forall \mathrm{v} \in \mathcal{V}_{k}, \forall k \in\{1,2, \ldots, K\} .
\end{gathered}
$$

where $\alpha_{1}, \alpha_{2}, \cdots, \alpha_{\infty}$ are the weights for neighbors at different steps. Note that introducing $\alpha_{n}$ in 19 does not violate the general form of Eq. (5) in Theorem 3.2 Let $\boldsymbol{B} \in\{0,1\}^{|\mathcal{V}| \times|\mathcal{V}|}$ be the adjacent matrix indicating all edges by 1 . We involve the polynomial matrix function on the adjacency matrix $B$ to represent the structures of various steps neighborhood with tuning the weights among 1-step to infinite-step neighbors automatically. If we define the polynomial matrix function $\Phi(\cdot)$ on the adjacent matrix $\boldsymbol{B}$ as $\Phi(\boldsymbol{B})=\sum_{n=0}^{\infty} \alpha_{n} \boldsymbol{B}^{n}$, we can cast Eq. (19) into its matrix form

$$
\begin{aligned}
\mathbf{x}^{\mathrm{v}}= & \mathbf{h}_{k}\left(\overline{\mathbf{g}}_{1}\left(\boldsymbol{X}_{1}\right)[\Phi(\boldsymbol{B})]_{\mathcal{V}_{1}, \mathrm{v}},\right. \\
& \overline{\mathbf{g}}_{2}\left(\boldsymbol{X}_{2}\right)[\Phi(\boldsymbol{B})] \mathcal{V}_{2, \mathrm{v}}, \cdots, \\
& \left.\mathbf{g}_{K}\left(\boldsymbol{X}_{K}\right)[\Phi(\boldsymbol{B})]_{\mathcal{V}_{K}, \mathrm{v}}\right), \quad \forall \mathrm{v} \in \mathcal{V}_{k}, \forall k \in\{1,2, \ldots, K\}
\end{aligned}
$$

where $\boldsymbol{X}_{k}$ denotes the representation matrix for nodes in type $k,[\Phi(\boldsymbol{B})]_{\mathcal{V}_{k}, \mathrm{v}}$ denotes the sub-matrix of $\Phi(\boldsymbol{B})$ indexed by column $\mathrm{v}$ and rows in $\mathcal{V}_{k}$, and function $\overline{\mathrm{g}}$ (with ${ }^{-}$on the top of function $\mathbf{g}(\cdot))$ is defined as the function extension

$$
\overline{\mathbf{g}}(\boldsymbol{X}):=\left[\mathbf{g}\left(\mathbf{x}_{1}\right), \mathbf{g}\left(\mathbf{x}_{2}\right), \cdots, \mathbf{g}\left(\mathbf{x}_{N}\right)\right]
$$

where $\overline{\mathbf{g}}(\cdot)$ takes the output column vectors from a function $\mathrm{g}(\cdot)$ with each column of $\boldsymbol{X}$ to form a new matrix with $N$ columns. For a specific node v, we apply the scale $\left|\mathcal{V}_{k, \mathrm{v}}\right|$ as $N$ for the type $k$ neighbors of node $\mathrm{v}$ in the equation above. Therefore, with the polynomial matrix function $\Phi(\boldsymbol{B})$ and multiple embedding vector mapping $\overline{\mathbf{g}}(\cdot)$ 's, we integrate the subtle structural knowledge of various types and steps neighbors surrounding a node to compute the node representation. Note that the embedding vectors for different types of nodes may be with different dimensions. Homogeneous graph is a special case of the heterogeneous graph with $K=1$. The above proposed model is thus naturally applicable to homogeneous graphs.

To avoid optimizing infinite number of coefficients, we propose to use a 1-dimensional NN function $\rho(\cdot): \mathbb{R} \mapsto \mathbb{R}$ to equivalently represent the function $\Phi(\cdot)$ to reduce the number of parameters based on the following observations

$$
\begin{aligned}
\Phi(\boldsymbol{B}) & =\boldsymbol{U} \operatorname{diag}\left(\sum_{n=0}^{\infty} \alpha_{n} \sigma_{1}^{n}, \cdots, \sum_{n=0}^{\infty} \alpha_{n} \sigma_{N}^{n}\right) \boldsymbol{U}^{\top} \\
& =\boldsymbol{U} \operatorname{diag}\left(\rho\left(\sigma_{1}\right), \cdots, \rho\left(\sigma_{N}\right)\right) \boldsymbol{U}^{\top},
\end{aligned}
$$

where $\boldsymbol{B}=\boldsymbol{U} \operatorname{diag}\left(\sigma_{1}, \cdots, \sigma_{N}\right) \boldsymbol{U}^{\top}$ is the singular value decomposition (SVD). We parameterize $\rho(\cdot)$ using 1dimensional NN function, which allows us easily controlling the number of variables to optimize in $\rho(\cdot)$ by choosing the number of layers and the number of nodes in each layer.

Remark B.1. Acute readers may worry about the computational complexity of SVD when the size of $\boldsymbol{B}$ is large. In general, the full SVD takes the cubic computational complexity $O\left(|\mathcal{V}|^{3}\right)$ where $|\mathcal{V}|$ is the total number of nodes in the network, which is pretty expensive if $|\mathcal{V}|$ is large. However, we can use the low-rank (top-r) singular value decomposition for approximation, where $r \ll|\mathcal{V}|$ is the target rank. Therefore, the computational complexity can reduce to $r^{2}|\mathcal{V}|$ from $|\mathcal{V}|^{3}$.

We can apply the low-rank approximation in the experiment for the node classification accuracy comparison. We compare the experiment results between the 1-step neighborhood PINE and multi-step neighborhood one. We present the results in Table 6 .

TABLE 6: 1-step v.s. Multi-step neighborhood

\begin{tabular}{c|cc}
\hline & PINE & Multi-step-P INE \\
\hline Cora & 85.58 & 85.13 \\
\hline Citeseer & 68.09 & 69.41 \\
\hline
\end{tabular}

